\title{
Clinical trials of Shigella vaccines: two steps forward and one step back on a long, hard road
}

\author{
Myron M. Levine, Karen L. Kotloff, Eileen M. Barry, Marcela F. Pasetti \\ and Marcelo B. Sztein
}

Abstract | More than 50 years of research has yielded numerous Shigella vaccine candidates that have exemplified both the promise of vaccine-induced prevention of shigellosis and the impediments to developing a safe and effective vaccine for widespread use, a goal that has yet to be attained. This Review discusses the most advanced strategies for Shigella vaccine development, the immune responses that are elicited following disease or vaccination, the factors that have accelerated or impeded Shigella vaccine development and our ideas for the way forward.

At the end of the 19th century, as epidemics of bacillary dysentery accompanied by high mortality spread across Japan, the young microbiologist Kiyoshi Shiga examined dysenteric stools and isolated a bacterium that was agglutinated by serum from convalescent patients but not from patients with acute disease ${ }^{1-3}$ (FIG. 1). That bacterium - known today as Shigella dysenteriae 1 - was the first identified member of the genus Shigella. Four Shigella species (or groups) are now recognized: S. dysenteriae (group A), which has 15 serotypes; Shigella flexneri (group B), which has 14 classical serotypes and subserotypes; Shigella boydii (group C), which has 20 serotypes; and Shigella sonnei (group D), which has a single serotype ${ }^{4}$ (TABLE 1).

There has been resurgent interest in Shigella as a human pathogen, driven by the availability of more precise data on the disease burden ${ }^{5-8}$, emerging antibiotic resistance ${ }^{9,10}$ and the fact that mucosally invasive Shigella, which often cause dysentery (gross blood in diarrhoeal stools), are less amenable to the salutary effects of oral rehydration than non-invasive pathogens that cause watery diarrhoea, such as Vibrio cholerae and enterotoxigenic Escherichia coli. The target populations for the use of Shigella vaccines include infants and young children in developing countries (in whom the peak incidence occurs at 12-47 months of age and the S. flexneri serotypes predominate $)^{5-7}$.

S. dysenteriae 1, which produces Shiga toxin and typically carries $\mathrm{R}$ factors that encode resistance to multiple antibiotics, waxes and wanes as a cause of epidemic severe disease in the world's least developed countries ${ }^{11-14}$.
Pandemics of Shiga dysentery, as occurred in Central America from 1968-1972 (REF. 11), South Asia in the $1970 \mathrm{~s}^{12}$, Central Africa in the $1980 \mathrm{~s}^{13}$ and East Africa in the $1990 \mathrm{~s}^{14,15}$ (FIG. 1), profoundly influence the global mortality burden that can be attributed to Shigella ${ }^{5,15}$. The lack of $S$. dysenteriae 1 endemicity results in low background immunity in populations, so epidemics of Shiga dysentery affect adults and children alike and the target ages for the use of a Shiga vaccine would be similarly broad ${ }^{1,11-13}$.

S. sonnei persists in developed (and transitional) countries, causing sporadic diarrhoea and occasional outbreaks in epidemiological niches (such as day-care centres) where personal hygiene can be suboptimal ${ }^{16,17}$. Travellers from developed to developing regions, who mainly acquire S. sonnei and S. flexneri infections ${ }^{18}$, represent another target population for Shigella vaccines. Shigellosis due to $S$. boydii or $S$. dysenteriae serotypes other than type 1 is uncommon ${ }^{5-7}$ (although increases in S. boydii have been reported in some foci ${ }^{7}$ ). Consequently, a Shigella vaccine that can provide a high level of protection against $S$. dysenteriae 1 , all S. flexneri serotypes and $S$. sonnei would constitute an epidemiologically valid 'global' vaccine ${ }^{5,19}$ (TABLE 1; BOX 1; FIG. 2).

Few bacterial pathogens have had their pathogenesis or interactions with mammalian tissues elucidated so precisely at the cellular and subcellular levels as Shigella spp. ${ }^{20,21}$ Nevertheless, progress in attaining safe and effective Shigella vaccines has faltered. Herein, we review recent and old clinical trials that have evaluated the safety, immunogenicity and efficacy of candidate Shigella vaccines. We relate the basis for the most popular 


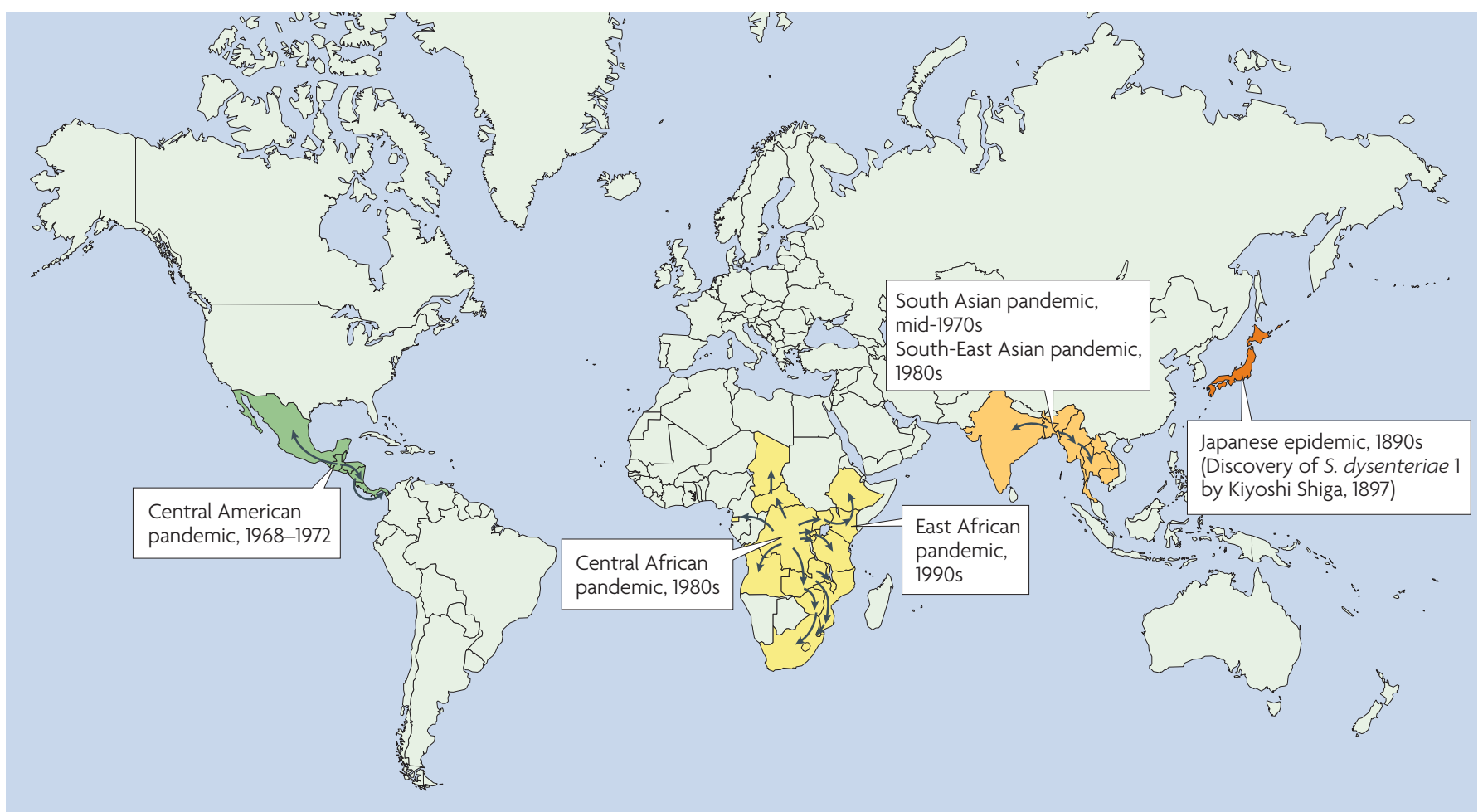

Figure 1 | Pandemics of Shiga dysentery. The first member of the genus Shigella to be identified, Shigella dysenteriae 1, was isolated by Kiyoshi Shiga during epidemics that occurred in Japan in the 1890s. Beginning in Central America in the late 1960s, pandemics of $S$. dysenteriae 1 caused severe disease with many complications and high case-fatality rates in all age groups. Subsequent pandemics occurred in Asia and Africa over the following 3 decades.

strategies (BOX 2), the relevance of the different immune responses measured, the factors that have favoured or impeded vaccine development and, most importantly, the lessons that must be learned for the future.

\section{Shigellosis models and vaccine development}

The ability of Shigella to cause diarrhoeal illness is restricted to human and higher non-human primate (NHP) hosts. Experimental models of shigellosis in adult human volunteers and NHPs have been invaluable for studying Shigella pathogenesis, infection and immunity as well as for evaluating candidate Shigella vaccines. Epidemiologically, shigellosis is a low-inoculum infection that is readily transmitted by direct faecal-oral contact. This has been corroborated by volunteer studies in which the ingestion of as few as ten S. dysenteriae 1 organisms induced a clinical infection rate of $10 \%^{22}$ and 200 organisms of different serotypes caused infection rates that were $>25 \%{ }^{22-26}$. In a volunteer model of experimental shigellosis that was developed in the late 1960s, the Shigella inoculum was administered in skimmed milk without buffer ${ }^{22,23,26,27}$. However, this was accompanied by considerable variability in the infection rate, particularly at lower inocula levels. A helpful modification was to administer the inoculum with $\mathrm{NaHCO}_{3}$ buffer ${ }^{25}$, which achieved consistency in the rate of shigellosis ${ }^{25,28}$ (TABLE 2). Volunteers develop classical bacillary dysentery, which commences with fever, malaise, abdominal cramps and (often watery) diarrhoea (for 18-24 hours). These symptoms are followed by scanty stools that contain blood and mucus and by tenesmus ${ }^{25}$.
With early antibiotic therapy, volunteers rapidly respond clinically and microbiologically (their cultures are often negative within 24 hours) $)^{25}$.

In contrast to the disease in humans, a large inoculum $\left(\sim 10^{10}\right.$ colony-forming units (CFU) $)$ administered with $\mathrm{NaHCO}_{3}$ is required to reliably induce shigellosis in NHPs ${ }^{29-31}$. So, although the NHP model is helpful for studying pathogenesis, infection-derived immunity and perhaps the efficacy of vaccines, it is not useful for predicting the clinical acceptability of putatively attenuated live vaccine candidates for humans.

The gross pathology of shigellosis in the human colon and the terminal ileum reflects the mucosal invasiveness of this pathogen. Understandably, most work on the molecular pathogenesis of Shigella infections has focused on the genes and gene products that are involved in invading epithelial cells and the host response to inva$\operatorname{sion}^{20,21}$. The early watery diarrhoeal phase of shigellosis was largely ignored until the NHP and volunteer model studies began to shed light on this stage of pathogenesis. Clinicians recognize that some patients with shigellosis manifest only watery diarrhoea, and even dysentery patients passing scanty bloody stools usually recount a day of watery diarrhoea without blood before the onset of their dysentery. When Rout et al. ${ }^{32}$ fed virulent S. flexneri 2a to NHPs, some NHPs developed only watery diarrhoea, whereas others developed only dysentery and some manifested both. In vivo perfusion studies revealed that colonic net transport of water occurred in all clinically ill NHPs, whereas only NHPs with watery 
diarrhoea exhibited net secretion of water, sodium and chloride ions in the jejunum, indicating the effect of an enterotoxin ${ }^{32}$. Histological examination demonstrated no jejunal abnormalities, corroborating the hypothesis that an enterotoxin (or toxins) was probably responsible for the watery diarrhoea. Kinsey et al. ${ }^{33}$ proved that the initiation of watery diarrhoea required that $S$. flexneri 2 a be present in the jejunum. When they bypassed the jejunum and inoculated NHPs directly in the cecum, the animals developed dysentery but not watery diarrhoea, and no jejunal secretion was observed ${ }^{33}$. The early watery diarrhoea phase, which can clearly be discerned in the volunteer model of shigellosis, prompted Fasano et al. to look for enterotoxins that could elicit small intestinal secretion in the absence of invasion ${ }^{34-37}$. Their efforts led to the discovery and characterization of Shigella enterotoxin 1 (ShET1; encoded by the chromosomal gene set) and ShET2 (encoded by the plasmid-borne gene sen) ${ }^{34-37}$. ShET1, a classical enterotoxin formed by a single A subunit that combines with five B subunits, is encoded on a chromosomal pathogenicity island ${ }^{38}$ and is restricted to isolates of $S$. flexneri $2 \mathrm{a}^{37-41}$. By contrast, ShET2, which is encoded by a gene on an invasiveness plasmid, is expressed by all Shigella serotypes ${ }^{36,39-41}$. The identification of these enterotoxins has had enormous repercussions for the development of well-tolerated live oral Shigella vaccine strains (TABLE 3).

\section{Immunity following wild-type infection}

Convincing evidence that an initial clinical Shigella infection elicits serotype-homologous protection comes from three sources: NHP challenge studies ${ }^{31}$, volunteer model re-challenge studies ${ }^{24,25,42}$ and prospective epidemiological surveillance of a cohort of children in an endemic area $^{6}$. Formal et al. reported that an initial S. flexneri $2 \mathrm{a}$ diarrhoeal infection in NHPs conferred $100 \%$ protection against re-challenge with $S$. flexneri 2 a, but offered no protection against challenge with $S$. sonnei $i^{31}$. For 30 months, Ferreccio et al. prospectively followed a cohort of children $<4$ years of age in a poor community in Santiago, Chile, where shigellosis was endemic. They found that 3 serotypes, S. sonnei, S. flexneri 2 a and S. flexneri 6, caused $79 \%$ of the cases of shigellosis ${ }^{6}$. As these serotypes do not share $\mathrm{O}$-antigen determinants (BOX 1), this provided a unique opportunity to investigate the immunity that follows natural clinical exposure to each of these serotypes. By comparing the incidence of subsequent episodes of shigellosis caused by a homologous serotype against the overall incidence of subsequent episodes, it was calculated that an initial bout of shigellosis afforded $72 \%$ protection against a second clinical illness due to the homologous serotype $(\mathrm{p}=0.05)^{6}$. Protection against a second clinical illness due to a heterologous serotype was low $(<30 \%$; $p>0.25)$. Collectively, these data suggest that antibodies to Shigella $\mathrm{O}$-antigen have a key role in protection (FIG. 3).

The debate over whether protection is predominantly mediated by mucosal secretory IgA (sIgA) anti-O-antigen antibodies, by serum IgG antibodies or by both (perhaps with contributions from other immune effector mechanisms) is more contentious. Strong mucosal (fae$\mathrm{cal}$ and urinary) sIgA anti-O-antigen antibody responses are observed following wild-type infection ${ }^{25,28,43-47}$ and experimental challenge ${ }^{25,28}$. Moreover, priming of the mucosal immune system following an initial wild-type Shigella infection has also been documented by enumerating gut-derived IgA anti-O-antigen antibody-secreting

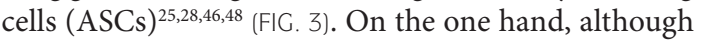
IgA anti-O-antigen ASCs and serum anti-O-antigen IgG have generally been considered key elements for protection, some studies have failed to show an association between these responses and resistance to illness following challenge $e^{49,50}$. In volunteer re-challenge studies, the substantial clinical protection exhibited by veterans ${ }^{24,25,42}$ is accompanied by an $\sim 1 \log$ reduction in the level of excretion of Shigella ${ }^{25}$. In one endemic situation, initial clinical infection by $S$. flexneri 2 a was followed by repetitive asymptomatic infections by that serotype and ultimately by sterile intestinal immunity such that even asymptomatic carriage was no longer detected $^{51}$. On the other hand, the importance of mucosal antibodies in preventing shigellosis was demonstrated by Tacket et al. who reported that passive oral administration of cow's milk immunoglobulin that contained high titres of anti-S. flexneri 2 a antibodies protected volunteers from experimental challenge with wild-type S. flexneri 2a strain $2457 \mathrm{~T}^{52}$. Notably, cow's milk immunoglobulin that contained low titres of antiS. flexneri $2 \mathrm{a}$ antibodies did not provide protection ${ }^{52}$. Additional research is needed to define the roles of intestinal sIgA and serum IgG antibodies in mediating protection against Shigella.

\section{Table 1 | Shigella species and serotypes}

\begin{tabular}{|c|c|c|c|}
\hline Shigella species (group) & $\begin{array}{l}\text { Number of serotypes } \\
\text { and subserotypes }\end{array}$ & Most important epidemiological niche & $\begin{array}{l}\text { Serotypes that a global vaccine } \\
\text { must protect against }\end{array}$ \\
\hline S. dysenteriae (group A) & 15 & $\begin{array}{l}\text { Epidemics and pandemics of severe Shiga } \\
\text { (S. dysenteriae 1) dysentery }\end{array}$ & S. dysenteriae 1 \\
\hline S. flexneri (group B) & $14^{*}$ & $\begin{array}{l}\text { Endemic paediatric shigellosis in developing } \\
\text { countries }\end{array}$ & All 14 types \\
\hline S. boydii (group C) & 20 & Scattered endemic foci in developing countries & None \\
\hline
\end{tabular}

*There are 14 classical Shigella flexneri serotypes and subserotypes ${ }^{4}$. 


\section{Box 1 | Shigella O-antigens}

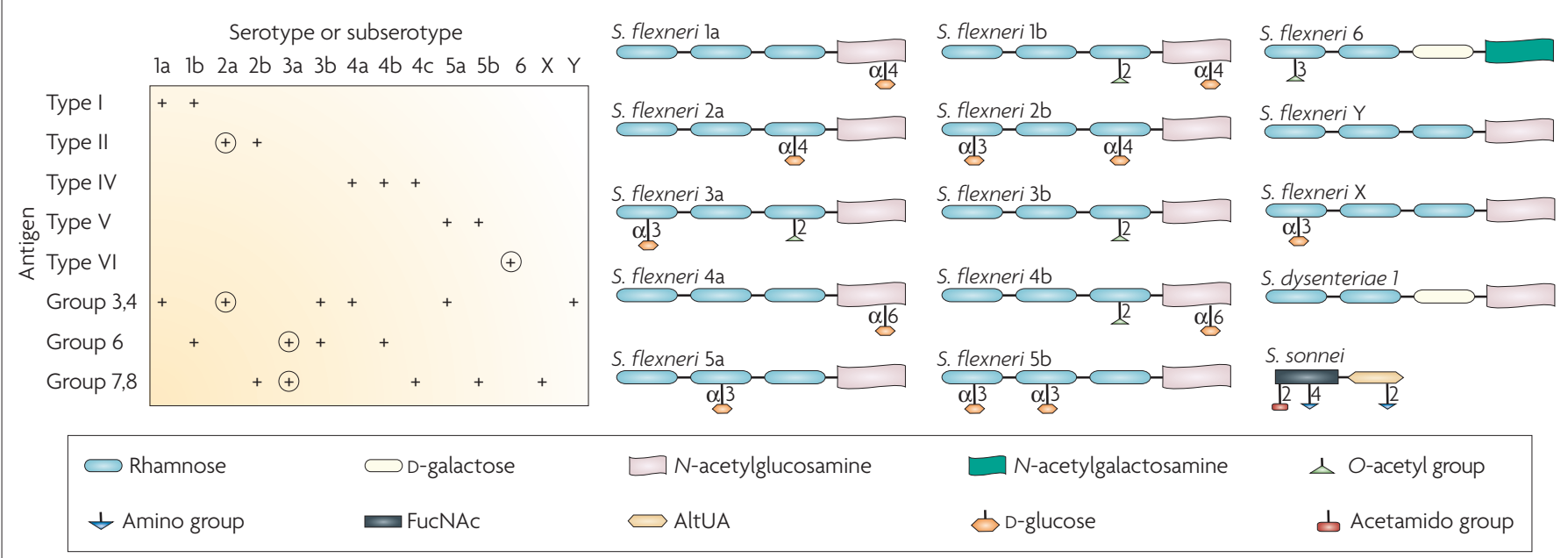

The lipopolysaccharide (LPS) of Shigella, a virulence factor ${ }^{119,120}$, consists of a toxic lipid A moiety embedded in the bacterial outer membrane, a core sugar region and an exposed terminal $O$ polysaccharide. Whereas there is little diversity in lipid $A$ and core regions among Shigella, the terminal O polysaccharide structures vary greatly, thereby giving rise to the immunological specificity that results in distinct serotypes (and subserotypes) 4 . Serotyping of Shigella flexneri is complicated. Current taxonomy recognizes 14 classical $S$. flexneri serotypes or subserotypes ${ }^{4}$, defined by type (I, II, IV-VI) and group (3, 4; $6 ; 7,8$ ) antigens (see left panel of the figure). Notably, the group antigens are shared among different serotypes. Type III antigen is not shown in the figure, as no structure can be assigned to it ${ }^{121}$. Moreover, absorbed rabbit 'type III' antisera actually bind group 6 antigen, indicating that type III antigen is identical to group 6 antigen, which is present in other serotypes ${ }^{121}$. Nevertheless, re-classification is unnecessary as strains heretofore characterized as S. flexneri 3a and $3 \mathrm{~b}$ can be readily identified with absorbed or monoclonal typing sera ${ }^{4,121,122}$; $\mathrm{S}$. flexneri $3 \mathrm{a}$ agglutinate with antisera to group 6 and 7,8 antigens and S. flexneri $3 b$ agglutinate with antisera to group 6 and 3,4 antigens (left panel) ${ }^{121}$.

Thirteen of the 14 classical S. flexneri serotypes or subserotypes (all except $S$. flexneri 6 ) share a common backbone of tetrasaccharide repeats that contain three rhamnose residues and one $\mathrm{N}$-acetylglucosamine (right panel). Genes that encode proteins involved in the synthesis of the linear backbone are located in the $r f b$ locus of the chromosome, whereas lysogenic phages encode the enzymes that link $\mathrm{O}$-acetyl groups or $\mathrm{D}$-glucose at various sites on the tetrasaccharide backbone, thereby creating or ablating antigenic epitopes. The resultant $\mathrm{O}$-antigens, the structures of which are displayed schematically in the right panel of the figure, provide the basis for the distinct S. flexneri serotypes or subserotypes. S. flexneri 6 has D-galactose as the third sugar of the tetrasaccharide and $\mathrm{N}$-acetylgalactosamine as the terminal residue (right panel). The structure of S. flexneri $4 \mathrm{c}$ is not shown as it has not been reported.

As Shigella dysenteriae 1 and Shigella sonnei are two serotypes that should be included in a global Shigella vaccine, the basic structures of their $\mathrm{O}$ polysaccharide repeats are also shown ${ }^{103,123}$. In contrast to the other $\mathrm{O}$-antigens that have tetrasaccharide repeats, the $\mathrm{O}$-antigen repeat of $S$. sonnei is a disaccharide (FucNAc, 2-acetamido-4-amino2,4,6-trideoxy-D-galactose; AltUA, 2-amino-2-deoxy-L-altruronic acid ${ }^{103}$. The $S$. dysenteriae $1 \mathrm{O}$ polysaccharide repeat most closely resembles the structures of S. flexneri 6 and S. flexneri $Y$ of the S. flexneri subtypes.

The left panel displays the $14 \mathrm{~S}$. flexneri serotypes and subserotypes along the horizontal axis and the type and group antigens in the vertical axis. Below each serotype or subserotype, the specific type and group antigens expressed are indicated by a plus sign. As the $\mathrm{O}$-antigens (circled) of S. flexneri 2a and S. flexneri 3a crossreact with 10 other S. flexneri serotypes or subserotypes, S. flexneri 2a, S. flexneri 3a and S. flexneri 6 have been proposed as three components of a pentavalent vaccine (along with S. dysenteriae 1 and S. sonnei) that could confer broad protection. Cross-protection among S. flexneri subserotypes has been demonstrated in a guinea pig model $^{118}$. A high priority is to test this strategy in humans.
T helper 1

( $T_{H} 1$ cell). A type of activated $\mathrm{T}_{\mathrm{H}}$ cell that promotes responses associated with the production of a particular set of cytokines, including interleukin-2 and interferon- $\gamma$, the main function of which is to stimulate phagocytosis-mediated defences against intracellular pathogens.
Other immune effector mechanisms have also been described in shigellosis (FIG. 3). Increases in serum antiIpa (invasion plasmid antigen) antibodies have been demonstrated after natural disease and experimental challenge ${ }^{25,43,53}$, and in endemic areas the seroprevalence of anti-Ipa antibodies increases with age ${ }^{43,54}$. A few studies have also measured cell-mediated immunity (CMI) elicited by wild-type Shigella infection. Rectal biopsies from Bangladeshis during acute and convalescent $S$. flexneri and $S$. dysenteriae 1 infection revealed an increased frequency of cytokine-producing cells and mononuclear cell infiltration in the rectal mucosa ${ }^{55}$. Increased levels of interferon- $\gamma($ IFN- $\gamma)$ in plasma and stool, upregulation of IFN- $\gamma$ receptor expression in patients with shigellosis and increased IFN- $\gamma$ production during convalescence suggest that this cytokine might play a role in both pathogenesis and protection ${ }^{55-59}$. Upon stimulation with Shigella antigens (including Ipa), the production of IFN- $\gamma$ and interleukin-10 (IL-10), but not IL-4, IL-5, IL-12 or IL-15, was demonstrated in peripheral blood mononuclear cells (PBMC) from volunteers challenged with modified virulent $S$. dysenteriae 1 strain SC595, indicating a predominant $\mathrm{T}$ helper $1\left(\mathrm{~T}_{\mathrm{H}} 1\right)$-type response ${ }^{60}$. Also, increased proportions of memory T cells $\left(\mathrm{CD} 45 \mathrm{RO}^{+}\right)$, the expression of T-cell activation molecules (for example, CD25, CD38, HLA-DR and CD54) and the expansion of defined $\mathrm{T}$-cell receptor $\mathrm{V} \beta$ families have been reported in patients with shigellosis ${ }^{61,62}$. Despite these findings, the contribution to protection from Shigella that can be attributed to CMI or to antibodies directed at Shigella proteins (such as Ipa and VirG) rather than O polysaccharide antigens remains controversial (FIG. 3). 


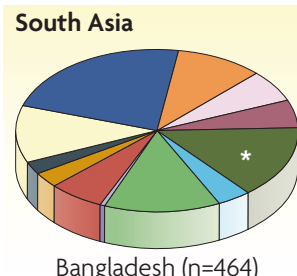

Bangladesh $(\mathrm{n}=464)$

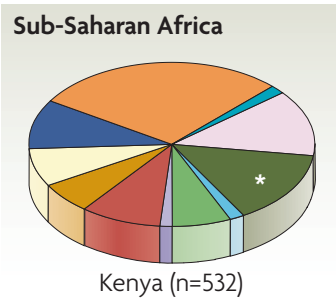

S. flexneri la

S. flexneri $1 \mathrm{~b}$

S. flexneri lc
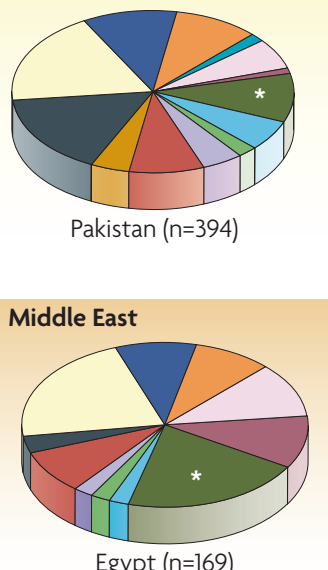

Egypt $(\mathrm{n}=169)$
East Asia and Pacific
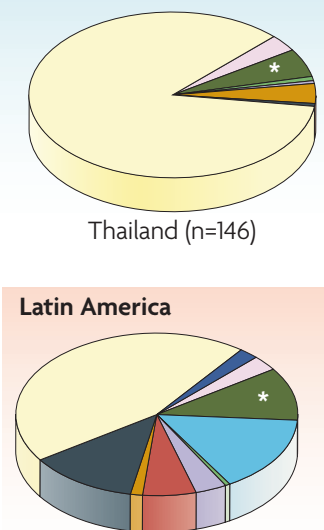

Chile (Santiago, Colina) (n=269)

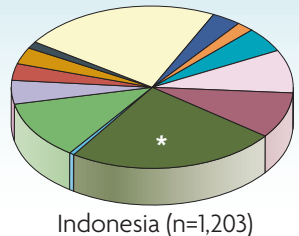

Indonesia $(\mathrm{n}=1,203)$

\begin{tabular}{|c|c|c|c|c|}
\hline S. flexneri $1 \mathrm{~b}$ & S. flexneri $2 b$ & S. flexneri 5a & S. sonnei & $\begin{array}{l}\text { S. flexneri } \\
\text { other types }\end{array}$ \\
\hline
\end{tabular}

Figure 2| The distribution of Shigella species and Shigella flexneri serotypes from selected studies in Latin America, Asia and Africa. Several population-based surveillance studies lasting at least 2 years used extensive serotyping methods (including either commercial absorbed animal sera or monoclonal antibodies for Shigella flexneri typing) ${ }^{6-9,122,129,130}$. In all countries, S. flexneri 2a (marked with an asterisk) is the single most important S. flexneri subserotype. The most developed of the countries, Chile and Thailand, show a notable proportion of cases due to Shigella sonnei. In any individual country, 3-4 serotypes constitute $75 \%$ of all cases. However, the other important S. flexneri serotypes, in addition to S. flexneri 2a, differ from country to country. Serotype $1 \mathrm{c}$ described in some studies is a provisional serotype. If the Center for Vaccine Development (CVD) pentavalent vaccine strategy that incorporates S. flexneri 2a, S. flexneri 3a and S. flexneri 6 (along with Shigella dysenteriae 1 and S. sonnei) succeeds in conferring cross-protection against the other 11 classical S. flexneri serotypes in future field trials, it should be possible to achieve a broad-spectrum global vaccine against shigellosis. S. dysenteriae type 1 were identified only in Kenya, where they comprised $67 \%$ of S. dysenteriae isolates. NT, non-typable.

\section{Protective prototype vaccines}

Two prototype Shigella vaccines, one which uses attenuated strains as live oral vaccines and the other which uses parenteral conjugates of Shigella O polysaccharide covalently linked to a carrier protein, have conferred significant protection in controlled Phase III trials. As these vaccines are furthest along in development, and as the results of the multiple clinical trials that have been carried out collectively provide insights, they will be discussed in depth. Other publications address promising alternative strategies (BOX 2) to develop Shigella vaccines ${ }^{63}$.

In the 1960s, Mel of the Military Medical Institute, Belgrade, Yugoslavia (Serbia), serially passaged different Shigella serotypes on streptomycin-containing media until they became streptomycin-resistant and streptomycin-dependent $(\mathrm{SmD}$; that is, they could not grow in the absence of exogenous streptomycin $)^{64,65}$. These SmD strains lost their ability to cause purulent keratoconjunctivitis in guinea pigs, a reliable test for Shigella mucosal invasiveness, intercellular spread and propensity to cause inflammation ${ }^{66}$. At the time, the genetic changes responsible for the attenuation were unknown. In addition to testing the vaccines in adults and children in Yugoslavia ${ }^{67-70}, \mathrm{SmD}$ Shigella vaccines were tested in adults and children in institutions in the United States where the risk of aquiring shigellosis was high ${ }^{42,51,71-73}$.
These vaccine strains proved to be well tolerated clinically when administered to adults, healthy children and even debilitated institutionalized children ${ }^{42,51,71-73}$, with the main adverse reaction being vomiting in a small percentage of recipients following administration of the

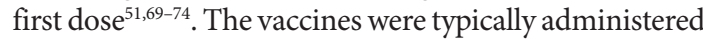
in an escalating $\left(2 \times 10^{10}, 3 \times 10^{10}, 4 \times 10^{10}\right.$ and $\left.5 \times 10^{10} \mathrm{CFU}\right)$ 4-dose regimen over 11 days. In controlled field trials, $\mathrm{Mel}$ et al. demonstrated the efficacy of the SmD vaccines $^{69,70}$, showed that multiple strains could be mixed together in combination vaccines and reported that protection was serotype-specific ${ }^{68-70}$. Protection endured for 1 year following primary immunization of children; however, administration of a single booster extended protection for an additional year ${ }^{70}$. These pioneering studies, which involved $\sim 36,000$ adult and paediatric subjects, provided proof of concept for future modern multivalent vaccines that aim to confer broad-spectrum protection. Unfortunately, the SmD vaccine strains had drawbacks that precluded them from becoming licensed for widespread use. In addition to the fact that the basis for the attenuation was unknown, occasional lots reverted to streptomycin independence (although the organisms remained unable to invade epithelium) and difficulties were encountered in large-scale manufacture and process control ${ }^{73,75}$. 


\section{Box 2 | Shigella vaccine strategies}

Strategies for which there is evidence from field trials that demonstrates efficacy: - Attenuated strains of Shigella used as live oral vaccines.

- O polysaccharides of Shigella covalently linked to carrier proteins and used as parenteral conjugate vaccines.

Strategies that are being (or have been) pursued but for which evidence of protection in humans has not been documented:

- Proteosomes (outer membrane vesicles of group B Neisseria meningitidis) to which Shigella sonnei or Shigella flexneri $2 \mathrm{a}$ is adsorbed ${ }^{124}$. This vaccine has been administered to humans intranasally.

- Inactivated S. sonnei administered orally ${ }^{125}$.

- Shigella invasion complex (Invaplex); a subcellular vaccine prepared from pathogenic S. flexneri, containing proteins including invasion plasmid antigen $\mathrm{B}$ (IpaB) and IpaC and lipopolysaccharide, administered to humans intranasally ${ }^{126}$.

- Nuclear protein-ribosomal parenteral vaccine prepared from a Shigella strain that has been genetically attenuated by an $m s b B$ mutation that partially detoxifies the lipid $A$ portion of the Shigella endotoxin ${ }^{127,128}$.
Phase I trial

Initial evaluation of the safety and immunogenicity of a vaccine in small groups of wise manner, receive increasing dosage levels of the vaccine. For live vaccines, vaccine shedding is also monitored. If the Phase I study is conducted under physical containment (such as in a research isolation ward), one can preliminarily assess the propensity of the live vaccine to be transmitted by including several placebo recipients as contact controls.

Phase II trial

Expanded (usually placebo controlled) studies of the safety, clinical acceptability and immunogenicity of a vaccine in larger numbers (often tens or hundreds) of subjects who exemplify the target age and other

characteristics for the use of the vaccine. Phase II trials aim to identify the optimal dosage level, formulation and

immunization regimen of the vaccine to be used in the

target groups, thereby paving the way for a pivotal Phase III efficacy trial. healthy subjects who, in a step-
S. flexneri $2 \mathrm{a}$ strain $\mathrm{T}_{32}$ is another pioneering vaccine strain developed by Istrati in Romania, where it was shown to be well tolerated and probably protective $^{76,77}$. Subsequently, in randomized placebo-controlled field trials in China, Bingrui et al. reported that $\mathrm{T}_{32}$ conferred significant protection against $S$. flexneri $2 \mathrm{a}$ diarrhoea $^{78}$. Surprisingly, the Chinese field trials also suggested that $\mathrm{T}_{32}$ conferred significant (albeit lower level) protection against shigellosis due to $S$. flexneri $1 \mathrm{~b}$ and $S$. boydii 1-6. Investigators at the Walter Reed Army Institute of Research (WRAIR) revealed that $\mathrm{T}_{32}$ harboured a large deletion in the invasiveness plasmid. This deletion resulted in the loss of three loci, ipaBCDA, invA and $\operatorname{vir} G$ (also known as icsA), which crippled the ability of this strain to invade epithelial cells ${ }^{79}$. Further investigation showed that the $\mathrm{T}_{32}$ plasmid deletion encompassed sen (which encodes ShET2); the chromosomal set gene (which encodes ShET1) remained intact ${ }^{39}$.

In S. sonnei, the $r f b$ locus, which encodes O-antigen, is located on the form I invasiveness plasmid. Investigators at the Lanzhou Institute of Biological Products introduced an S. sonnei form I plasmid with deletions of ipa and virF into $S$. flexneri $2 \mathrm{a} \mathrm{T}_{32}$ (REF. 80). Three doses (from $2 \times 10^{10}$ to $5 \times 10^{10} \mathrm{CFU}$ ) of the resultant hybrid strain expressing both $S$. flexneri 2 a and $S$. sonnei $\mathrm{O}$-antigens provided $\sim 65 \%$ protection against both $S$. flexneri 2 a and $S$. sonnei diarrhoea ${ }^{80}$.
Formal et al. at WRAIR prepared a 'mutant-hybrid' $S$. flexneri 2 a vaccine strain by selecting a colonial mutant that had lost its ability to invade intestinal epithelial cells ${ }^{81}$ and transferred into it the xyloserhamnose region of $E$. coli (which diminishes the ability of Shigella to propagate in the lamina propria even if epithelial invasion occurs) ${ }^{82}$. In Phase I safety and Phase II immunogenicity clinical trials in adults and children in the United States ${ }^{71,72}$, the S. flexneri 2a mutant-hybrid vaccine was as well tolerated as Mel's SmD vaccines and conferred partial (albeit non-significant) protection against experimental challenge in adult volunteers ${ }^{42}$; by comparison, SmD S. flexneri 2 a conferred significant protection $^{42}$.

Building on the pioneering work of $\mathrm{Mel}$ and Istrati, researchers worldwide have used recombinant DNA technology and knowledge of the molecular pathogenesis of shigellosis to create modern live oral Shigella vaccine candidates that can overcome the drawbacks of the early strains. Progress has been steady but painstakingly slow, as unexpected obstacles have been encountered.

\section{The importance of the parent strain}

One lesson that has been learned in developing attenuated Shigella vaccines is the importance of starting with a wild-type parent strain of known virulence, ideally as assessed in volunteer studies. The paradigm is the array of derivatives of wild-type $S$. flexneri 2a strain 2457T, a parent strain known to be virulent in North American volunteers and to cause diarrhoea, fever and dysentery with an infection rate that increases with escalating doses ${ }^{25,49,50}$ (TABLE 2). Moreover, an initial clinical episode of 2457T-induced shigellosis stimulates immunity that confers $\sim 64-75 \%$ protection against illness upon subsequent re-challenge with this strain $^{25,42}$. As the baseline virulence of the parent strain is well documented, the degree of attenuation achieved by putative attenuating mutations can be quantified (TABLES 2,3).

It is difficult, however, to draw conclusions about the ability of specific mutations to cause attenuation when they are introduced into wild-type parent strains that were either not tested clinically beforehand or, if tested, were found to be only minimally pathogenic in humans. Two illustrative examples are wild-type S. flexneri Y strain SF1 and a Shiga-toxin-deleted derivative of wild-type S. dysenteriae 1 strain SC595 (which retains
Table 2 | Response to wild-type Shigella flexneri 2a

\begin{tabular}{|c|c|c|c|c|c|c|}
\hline \multirow[t]{2}{*}{ Challenge } & \multicolumn{3}{|c|}{ Adverse clinical outcomes } & \multicolumn{2}{|c|}{ IgA anti-O-antigen ASC } & \multirow[t]{2}{*}{ Refs } \\
\hline & Diarrhoea & Dysentery & Fever & \% Responders & Geometric mean* & \\
\hline Challenge no. 1 & $\begin{array}{l}12 / 14 \\
(86 \%)\end{array}$ & $\begin{array}{l}10 / 14 \\
(71 \%)\end{array}$ & $\begin{array}{l}10 / 14 \\
(71 \%)\end{array}$ & $100 \%$ & 143 & 50 \\
\hline Challenge no. 2 & $\begin{array}{l}11 / 12 \\
(92 \%)\end{array}$ & $\begin{array}{l}10 / 12 \\
(83 \%)\end{array}$ & $\begin{array}{l}10 / 12 \\
(83 \%)\end{array}$ & $92 \%$ & 239 & 25 \\
\hline
\end{tabular}

This Table shows the consistency of the clinical and immunological response in North American adult volunteers following challenge with $10^{3}$ colony-forming units (CFU) of wild-type Shigella flexneri 2a strain $2457 \mathrm{~T}$ administered with $\mathrm{NaHCO}_{3}$ buffer. ${ }^{*}$ Per $10^{6}$ PBMC. ASC, antibody-secreting cells; PBMC, peripheral blood mononuclear cells. 
Table 3 | The role of the ShET1 and ShET2 enterotoxins

\begin{tabular}{|c|c|c|c|c|c|c|c|c|}
\hline \multirow[t]{3}{*}{ Strain } & \multicolumn{6}{|c|}{ Adverse clinical outcomes } & \multicolumn{2}{|c|}{ IgA anti-O-antigen ASC } \\
\hline & \multicolumn{2}{|c|}{ Diarrhoea } & \multicolumn{2}{|c|}{ Dysentery } & \multicolumn{2}{|c|}{ Fever } & \multirow{2}{*}{$\begin{array}{l}\text { \% Responders } \\
10^{9}\end{array}$} & \multirow{2}{*}{$\begin{array}{l}\text { Geometric mean* } \\
\text { N/A }\end{array}$} \\
\hline & $10^{8}$ & $10^{9}$ & $10^{8}$ & $10^{9}$ & $10^{8}$ & $10^{9}$ & & \\
\hline$\triangle g u a B A(C V D 1204)$ & $\begin{array}{l}2 / 7 \\
(29 \%)\end{array}$ & $\begin{array}{l}4 / 7 \\
(57 \%)\end{array}$ & $0 / 7$ & $0 / 7$ & $0 / 7$ & $\begin{array}{l}4 / 7 \\
(57 \%)\end{array}$ & $100 \%$ & 445 \\
\hline $\begin{array}{l}\Delta \text { guaBA, } \Delta \text { set, } \\
\Delta \text { sen (CVD 1208) }\end{array}$ & $0 / 7$ & $0 / 7$ & $0 / 7$ & $0 / 7$ & $0 / 7$ & $\begin{array}{l}1 / 7 \\
(14 \%)\end{array}$ & $86 \%$ & 62 \\
\hline
\end{tabular}

This Table shows the consistency of the clinical and immunological response in North American adult volunteers to ingestion of $10^{8}$ or $10^{9}$ colony-forming units (CFU) of two deletion mutants of wild-type S. flexneri 2a strain $2457 \mathrm{~T}$ with $\mathrm{NaHCO}_{3}$ buffer $^{88}$. ${ }^{*}$ Per $10^{6}$ PBMC. ASC, antibody-secreting cells; PBMC, peripheral blood mononulcear cells; ShET, Shigella enterotoxin.

epithelial cell invasiveness and causes keratoconjunctivitis in guinea pigs). In the case of the S. flexneri Y strain $\mathrm{SF} 1$, an $\operatorname{aroD}$ mutation was introduced, resulting in attenuated putative vaccine candidate SFL124 (REF. 83). Subsequently, however, when the wild-type S. flexneri Y parent strain SF1 was fed to North American volunteers, it was only minimally pathogenic ${ }^{84}$. So, it was not possible to attribute the clinical behaviour of the vaccine strain to the introduced mutation. By contrast, when an aroD deletion was introduced into the well-characterized wild-type S. flexneri 2a strain $2457 \mathrm{~T}$, resulting in vaccine strain SFL1070, it was clearly demonstrated that this mutation afforded measurable attenuation ${ }^{85}$. Similarly, S. dysenteriae 1 strain SC595 caused only low rates of mild diarrhoeal illness when administered to North American volunteers (except in one subject who developed dysentery $)^{60}$. SC595 did, however, elicit robust antibody and CMI responses following ingestion of as few as $300 \mathrm{CFU}^{60}$. Not surprisingly, SC599, a derivative of SC595 that contains deletions in ics A (also known as $v i r G$; this deletion diminishes the intracellular and intercellular spread of Shigella), ent and fep (which encode proteins involved in iron chelation) was well tolerated in Phase I safety trials ${ }^{63}$. However, SC599 was only minimally excreted and induced only modest anti-O-antigen ASC responses and few anti-O-antigen seroconversions ${ }^{63}$.

\section{Selection of attenuating mutations}

The phenotypic consequences of introducing different mutations into wild-type $S$. flexneri 2a strains have been evaluated in Phase I clinical trials. These mutations include $\operatorname{aro} D$ and $\operatorname{aro} A$ (which encode enzymes in the aromatic amino-acid biosynthesis pathway; mutation confers auxotrophy for substrates not found in sufficient concentration in mammalian tissues $)^{85,86}, \operatorname{vir}^{28,87}$, iuc (which encodes aerobactin) ${ }^{28}$, guaBA (which encodes enzymes involved in guanine-nucleotide biosynthesis) ${ }^{87,88}$, set (which encodes ShET1) and sen (which encodes ShET2 $)^{87,88}$.

The experience with strain SC602, a derivative of wild-type strain Pasteur Institute S. flexneri 2a 494 with mutations in $v i r G$ and $i u c$, is instructive ${ }^{28,89}$. When fed to North American volunteers, dosages $\geq 10^{6} \mathrm{CFU}$ were unacceptably reactogenic, with most subjects developing diarrhoea, fever or severe constitutional symptoms, although the vaccine strain was heavily shed and highly immunogenic ${ }^{28}$. By contrast, at a dosage level of $10^{4} \mathrm{CFU}$, adverse clinical reactions were uncommon and mild, yet the induced immune response remained moderately robust ${ }^{28}$. Eight weeks after vaccinees received a single $10^{4} \mathrm{CFU}$ dose of SC602, 7 vaccinees (and 7 unvaccinated control volunteers) participated in an experimental challenge study and ingested $10^{3} \mathrm{CFU}$ of virulent $S$. flexneri 2 a strain $2457 \mathrm{~T}^{28}$. Diarrhoea was observed in $86 \%$ of the controls and in $43 \%$ of the vaccinees $(p=0.27)^{28}$. However, the 3 vaccinees that developed diarrhoea had significantly milder illness than the controls. Four of the 7 controls (57\%) developed dysentery, 6 (86\%) had fevers (mean temperature $39^{\circ} \mathrm{C}$ ) and $6(86 \%)$ manifested severe shigellosis. By contrast, none of the vaccinees had dysentery, fever or severe illness $(\mathrm{p}=0.0027)^{28}$.

This hallmark study showed that in the experimental challenge model even a single dose of an engineered vaccine strain could confer significant protection against severe shigellosis. However, it also demonstrated the difficulty of finding a proper balance between clinical acceptability and immunogenicity in adult volunteers in developed countries. Indeed, additional studies with SC602 in 34 community volunteers showed that $10^{4} \mathrm{CFU}$ caused mild diarrhoea in 5 volunteers (15\%) and fever in 4 volunteers $(12 \%)^{89}$.

Invaluable lessons have also been learnt from clinical trials with the series of attenuated derivatives of wild-type S. flexneri 2a strain 2457T that was developed by the University of Maryland Center for Vaccine Development (CVD), as the results have directed iterations in vaccine design. CVD 1203 is a derivative of $2457 \mathrm{~T}$ that harbours deletions in aro $A$ and $\operatorname{vir} G^{90}$. In a Phase I clinical trial in 10 North American adults, CVD 1203 was well tolerated at a dose of $10^{6} \mathrm{CFU}$ (a dosage level at which SC602 was extremely reactogenic) ${ }^{86}$. However, when administered at doses of $10^{8} \mathrm{CFU}$ or $10^{9} \mathrm{CFU}^{86}$, CVD 1203 induced unacceptable reactogenicity accompanied by strong immune responses ${ }^{86}$. Significant increases in the concentrations of tumour-necrosis factor- $\alpha$ (TNF- $\alpha$ ) were observed in serum and stool, suggesting that this cytokine might be related to the reactogenicity observed at high doses ${ }^{86}$. Although the potent immune responses to CVD 1203 were encouraging, the reactogenicity dictated that alternative mutations should be evaluated to achieve a satisfactory degree of attenuation. 
a Wild-type infection

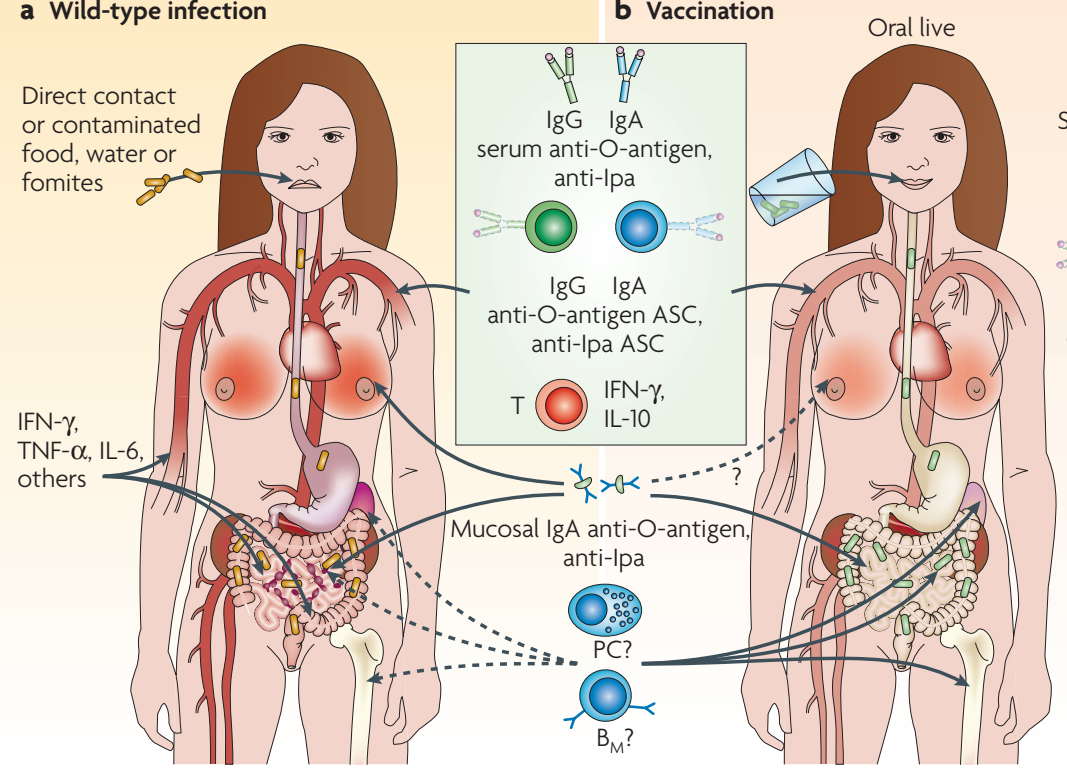

Inductive site of

immune response

Serum antibodies

Mucosal

antibodies

Circulating ASC

CMI

Protection (field studies)

Protection GALT

GALT

$\lg \mathrm{G}$, IgA anti-O-antigen, anti-Ipa, other antigens?

Breast milk: slgA anti-O-antigen, anti-Ipa Intestinal: slgA anti-O-antigen, anti-Ipa

Gut-derived ASC: IgA, IgG anti-O-antigen, anti-Ipa, other antigens?

Proliferation

$\mathrm{T}_{\mathrm{H}}$ 1-type cytokines (IFN- $\gamma$ ), IL-10

(challenge studies)
Yes (children)

Yes (adults) $\lg \mathrm{G}, \lg \mathrm{A}, \lg \mathrm{M}$ (low) anti-O-antigen, antiIpa, other antigens?

Intestinal: slgA anti-O-antigen, anti-lpa, other antigens?

Gut-derived ASC: IgA, IgG anti-Oantigen, anti-Ipa, other antigens?

$\mathrm{T}_{\mathrm{H}}$ 1-type cytokines (IFN- $\gamma$ ), IL-10

Yes (children and adults)

Yes (adults)

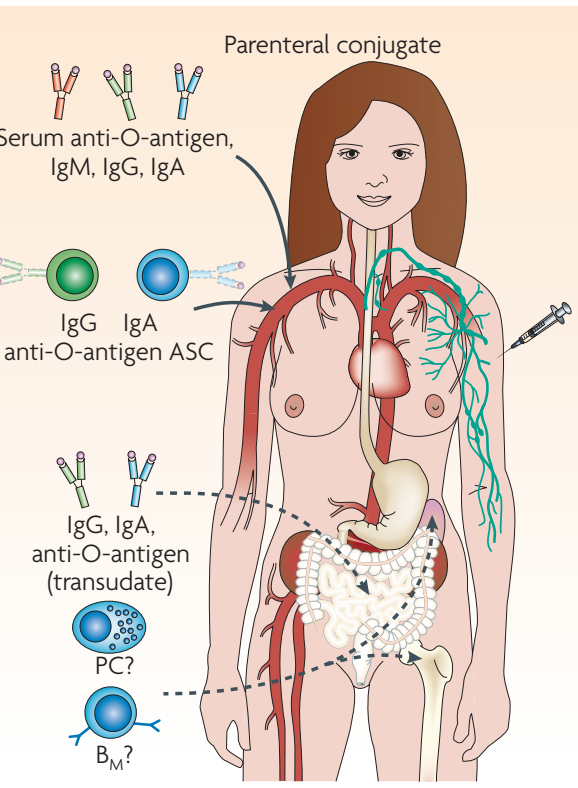

Peripheral lymph nodes

$\lg \mathrm{G}, \lg \mathrm{A}, \lg \mathrm{M}$ anti-O-antigen

Intestinal: $\lg G$ (serum transudate) anti-O-antigen

Urinary: slgA anti-O-antigen

Secondary lymphoid organ-derived ASC: $\lg A$, lgG anti-O-antigen

ND

Yes (adults)

ND

Figure 3 | Immunity to Shigella. a | Immune responses to wild-type Shigella infection. The specific immune responses that mediate protection against shigellosis remain controversial. The strong serum $\lg G$ and $\lg A$ antibody responses to Shigella O-antigen induced by wild-type infection correlate with protection data from epidemiological ${ }^{6}$ and seroepidemiological studies ${ }^{11,131}$ as well as non-human primate challenges ${ }^{31}$. Gut-derived IgA anti-O-antigen antibodysecreting cell (ASC) responses are also thought to have a significant role in protection. By contrast, the role of antibody responses to Shigella protein antigens (such as invasion plasmid antigen (lpa) proteins, VirG and others) and of cellmediated immunity (CMI) in contributing to protection remain a matter of speculation. $\mathbf{b}$ | Immune responses to vaccines. The immune responses generated by types of Shigella vaccines (live oral and parenteral conjugate) that have been shown to confer protection are depicted. Responses elicited by live oral vaccines include $\lg \mathrm{A}$ and $\lg \mathrm{G}$ anti-O-antigen and anti-Ipa ASC detected among peripheral blood mononuclear cells (PBMC), serum IgA and IgG anti-O-antigen and anti-Ipa, as well as IgA anti-O-antigen in stool, intestinal fluid and urine ${ }^{25,49,50,60,86}$. Interferon- $\gamma$ (IFN- $\gamma$ ) responses to Shigella antigens have also been observed in volunteers immunized with Shigella flexneri 2a strain CVD 1207 (REF. 87), Shigella sonnei strain WRSS1 (REF. 94) and SFL1070 (REF. 85). The contribution of CMI and of antibodies to Shigella proteins (for example, Ipa and $\mathrm{VirG}$ ) elicited by live oral vaccines in preventing clinical Shigella infection remains uncertain. In contrast to the broad immune responses elicited by live oral vaccines, parenteral conjugate vaccines elicit a narrower response, characterized predominantly by serum IgG antibodies to Shigella O-antigen. Wild-type Shigella or live oral vaccines are likely to generate long- and short-lived plasma cells $(\mathrm{PC})$ and memory $B$ cells $\left(\mathrm{B}_{\mathrm{M}}\right)$ that reside in the bone marrow, spleen and gutassociated lymphoid tissue (GALT). By contrast, $P C$ and $B_{M}$ elicited by conjugate vaccines are likely to be confined to the bone marrow and spleen. The relative magnitudes of immune responses are depicted by gradients of colour at the various effector sites. IL, interleukin; ND, no data; TNF- $\alpha$, tumour-necrosis factor- $\alpha$.

Strain CVD 1207 harbours deletion mutations in $g u a B A^{91}, \operatorname{vir}^{90}, \operatorname{set}^{34}$ and $\operatorname{sen}^{36}$. When volunteers ingested escalating single doses of up to $10^{10} \mathrm{CFU}$ of CVD 1207 (REF. 87), this live vaccine strain was remarkably well tolerated. No subjects manifested adverse reactions through the $10^{8} \mathrm{CFU}$ dosage level ${ }^{87}$, and even at $10^{9}$ and $10^{10} \mathrm{CFU}$ only a few volunteers experienced mild diarrhoea ${ }^{87}$. The conclusion from the Phase I clinical trial of CVD 1207 was that the mutations definitively attenuated the $2457 \mathrm{~T}$ parent strain, resulting in a vaccine that was 
well tolerated by North American volunteers at high dosage levels yet remained immunogenic. However, it was hypothesized that strain CVD 1207 could have been hyperattenuated ${ }^{87}$.

CVD 1204 and CVD 1208, isogenic derivatives of wild-type strain $2457 \mathrm{~T}$, both carry deletions in guaBA ${ }^{88,91}$, and CVD 1208 also has deletions in sen and set. These strains differ from isogenic CVD 1207 by having an intact $\operatorname{vir} G$ gene. This design permitted researchers to assess the impact of inactivating ShET1 and ShET2 (TABLE 3). A randomized, doubleblind clinical trial was performed in adult North Americans who ingested a single dose containing $10^{7}$, $10^{8}$ or $10^{9} \mathrm{CFU}$ of either strain ${ }^{88}$. Objective adverse reactions were observed in 35\% of CVD 1204 recipients but only in $4 \%$ of CVD 1208 recipients $(\mathrm{p}=0.02)^{88}$. Immune responses to $S$. flexneri 2 a O-antigen (ASCs, serum IgG or faecal IgA) were observed in $86 \%, 43 \%$ and $100 \%$ of CVD 1208 recipients, respectively. The enterotoxin-negative strain CVD 1208 was considered a highly attractive vaccine candidate that reflects the desired balance of clinical acceptability and robust immunogenicity (TABLE 3). As this strain was constructed using culture media that contained animal products, it was reconstructed using soy-based media for regulatory purposes, resulting in strain CVD 1208S. In a Phase I clinical trial, CVD 1208 S demonstrated the same clinical acceptability, shedding pattern and immunogenicity as CVD 1208 (K.L.K., unpublished data). Thus, CVD 1208S, like CVD 1208, exhibits the desirable balance of low reactogenicity and robust immunogenicity.

Investigators at WRAIR constructed strain WRSS1 by deleting virG in the wild-type $S$. sonnei Moseley strain $^{92}$. Although the virulence of this $S$. sonnei strain had not been demonstrated in volunteers, it was selected because its form I invasiveness plasmid (required for expression of $\mathrm{O}$-antigen by this serotype) was remarkably stable, in contrast with the form I plasmid of most wild-type S. sonnei strains ${ }^{24,93}$. In a Phase I trial in North American volunteers given a single dose of up to $10^{6} \mathrm{CFU}$, low-grade fever or mild diarrhoea was recorded in 6 of 27 (22\%) WRSS1 vaccinees ${ }^{94}$. Strong IgA anti-O-antigen ASC responses were observed in recipients of all dosage levels ${ }^{94}$. Moderate IFN- $\gamma$ responses were also observed in some volunteers ${ }^{94}$.

Safety and immunogenicity trials of WRSS1 were next performed in Israel in community adults who ingested $10^{3}, 10^{4}$ or $10^{5} \mathrm{CFU}^{95}$. At the two lower dosage levels, the vaccine was well tolerated with the exception of 1 of 30 subjects (3\%) who developed moderate diarrhoea, and 5 (17\%) who experienced mild diarrhoea. By contrast, at the $10^{5} \mathrm{CFU}$ dosage level, 2 of 15 subjects (13\%) developed fever and $4(27 \%)$ experienced moderate diarrhoea ${ }^{95}$. The $10^{4} \mathrm{CFU}$ dosage level provided the better balance of immunogenicity and clinical acceptability, as all subjects manifested IgA anti-O-antigen ASC responses and $73 \%$ of the vaccinees showed more than $50 \mathrm{IgA}$ anti-O-antigen ASCs per $10^{6} \mathrm{PBMC}^{95}$.
So, certain attenuating mutations introduced into wild-type strains in recent years have generated promising vaccine candidates. Some modifications, for example deletion of the genes that encode ShET1 and ShET2, might work well in combination with an array of other attenuating mutations. If commercial and intellectual property considerations do not create barriers, it would be of interest to 'mix and match' novel combinations of attenuating mutations that have not previously been used in the same strains. For example, in their latest generation of live vaccine candidates, WRAIR investigators have deleted the ShET-encoding genes in strains that carry a $\operatorname{vir} G$ deletion as the primary attenuating mutation (M. Venketasan, personal communication). Similarly, it would be instructive to directly compare the clinical acceptability and immunogenicity of two or more candidate live vaccine strains of the same serotype in randomized, double-blind clinical trials. Last, it has generally been proposed that once attenuating mutations have been documented in one serotype, they can be introduced into other epidemiologically relevant serotypes. However, if the commercial considerations can be managed, another track towards licensure of a broadly protective vaccine would be to combine promising strains of several different serotypes that have been attenuated by distinct mutations.

\section{Hybrid live vector Shigella vaccines}

As an alternative strategy to construct live Shigella vaccines, several research groups have attempted to express Shigella O- and other (Ipa) antigens in welltolerated live vectors such as normal flora, rough E. coli or highly attenuated Salmonella enterica serovar Typhi. The genes that allow synthesis of group- and type-specific O-antigens of $S$. flexneri 2 a were introduced into an E. coli $\mathrm{O} 8$ strain, resulting in the vaccine strain PGAI 42-1-15. This hybrid E. coli expressing S. flexneri 2a $\mathrm{O}$-antigen was well tolerated when given to adult US volunteers and was excreted for several days ${ }^{27}$. In two separate large experimental challenge studies, volunteers given two (first study) or three (second study) doses of vaccine one month apart were challenged 4-8 weeks later with $10^{4} \mathrm{CFU}$ (first study) or $10^{2} \mathrm{CFU}$ (second study) of virulent $S$. flexneri 2 a strain $2457 \mathrm{~T}^{27}$. The rates of shigellosis were similar in vaccinees and controls ${ }^{27}$. It was unclear why this live vaccine failed to protect. However, one hypothesis is that to be protective, an E. coli live vector strain expressing Shigella $\mathrm{O}$-antigens must also exhibit the capacity to invade epithelial cells ${ }^{27}$. Accordingly, investigators at WRAIR constructed two E. coli live vector strains expressing S. flexneri 2a O-antigen that manifested epithelial cell invasiveness.

The invasiveness plasmid of $S$. flexneri 5 and the genes that allow expression of $S$. flexneri 2a type and group-specific $\mathrm{O}$-antigens were introduced into E. coli $\mathrm{K} 12$, producing a strain, EcSf2a-1, that could invade epithelial cells ${ }^{49}$. At a dose of $10^{9} \mathrm{CFU}$, this strain was unacceptably reactogenic as 4 of 13 (31\%) vaccinees developed fever, diarrhoea or dysentery. 
However, at lower, better-tolerated dosage levels, the vaccine failed to protect vaccinees against experimental challenge with $S$. flexneri $2 \mathrm{a}^{49}$. In an attempt to diminish reactogenicity, an aroD deletion was introduced, resulting in the further derivative strain EcSf2a-2 (REF. 96). At high dosage levels, this strain, which retained the ability to invade epithelial cells, also caused adverse reactions but was strongly immunogenic. However, despite robust immunogenicity, the vaccine conferred only $36 \%$ protection against illness (fever, diarrhoea or dysentery) upon experimental challenge $(\mathrm{p}=0.17)^{50}$. Overall, E. coli, with or without epithelial invasiveness, has been unsuccessful as a live vector vaccine expressing Shigella $\mathrm{O}$-antigen.

In the 1980s, Formal and co-workers at WRAIR developed a vaccine candidate that consisted of licensed oral Salmonella Typhi live vaccine strain Ty21a engineered to express the O polysaccharide of $S$. sonne $i^{97}$. This live vector strain, 5076-1C, was well tolerated but exhibited lot-to-lot variability in its immunogenicity and ability to protect in challenge studies in which vaccinees and controls ingested wild-type $S$. sonnei strain $53 \mathrm{G}^{24,48,98}$; however, two pilot lots did confer significant protection ${ }^{93}$. Multiple hypotheses were pursued to explain the lot-to-lot variability in efficacy ${ }^{99-102}$, but none were predictive and further development of 5076-1C was abandoned ${ }^{24}$.

To overcome the inconsistency of 5076-1C, Kopecko and co-workers attempted to more reliably express S. sonnei $\mathrm{O}$-antigen in Ty21a, as well as $\mathrm{O}$-antigens from other serotypes ${ }^{103}$. As Salmonella has a lipopolysaccharide (LPS) core that is chemically distinct from that of Shigella ${ }^{103}$, S. sonnei O polysaccharide in Salmonella Typhi is expressed only as a non-core-linked surface-associated antigen ${ }^{103}$. Clinical trials are required to assess if these new Ty21a-based constructs will offer more consistent protection.

\section{Intestinal barrier in developing countries}

Oral vaccines against polio, rotavirus and cholera have exhibited lower immunogenicity in underprivileged subjects in developing countries compared with subjects in developed countries ${ }^{104-107}$. The immunogenicity of some of these vaccines was successfully enhanced by increasing the number of vaccine organisms per dose or by administering additional doses of vaccine $e^{104,106}$. Although S. flexneri 2a vaccine strain SC602 had been reactogenic in North American adults, as it conferred protection against severe disease in an experimental challenge, Phase I and II trials were undertaken in adults and children in a developing country. None of 68 Bangladeshi adults who ingested up to $10^{6} \mathrm{CFU}$ of SC602 experienced adverse reactions, but neither did any vaccinee shed SC602, and the immune responses induced were meager ${ }^{63}$. Next, Bangladeshi children of 8-10 years of age and 12-36 months of age ingested up to $10^{6} \mathrm{CFU}^{63}$. Although no child manifested an adverse reaction, neither did any child excrete the vaccine strain or mount a significant immune response. So, the developing country subjects manifested markedly different clinical, bacteriological and immunological responses to SC602 compared with North American subjects. In adults in the United States, a low dose $\left(10^{4} \mathrm{CFU}\right)$ of SC602 was reactogenic, heavily excreted and moderately immunogenic, whereas in the children and adults in Bangladesh it was virtually inert, even in a 100-fold higher $\operatorname{dose}^{63}$. One possible hypothesis to explain these divergent results is that Bangladeshis have decreased levels of accessible iron in tissues compared with subjects in developed countries, so that SC602 is more crippled in individuals in developing countries.

Does this mean that most live oral Shigella vaccines will be poorly immunogenic in developing country populations? The response of Vietnamese adults ${ }^{83}$ and children ${ }^{108}$ to the ingestion of $S$. flexneri Y strain SFL124 (an aroD-deletion mutant) and the efficacy of the SmD and T32 live oral Shigella vaccines in subjects in Yugoslavia (late 1960s and early 1970s) (99,70 $^{6}$ and China $(1980 \mathrm{~s})^{78}$, respectively, suggest not. A single $2 \times 10^{9} \mathrm{CFU}$ dose of SFL124 was administered to 15 Vietnamese adult volunteers, whereas another 15 subjects ingested three doses (at an interval of two days between doses) ${ }^{83}$. No subjects manifested fever or diarrhoea, SFL124 was excreted by all volunteers and was immunogenic. Although few subjects excreted the strain, SFL124 was also immunogenic in Vietnamese children of 9-14 years of age and stimulated local mucosal immune responses and ASC responses to $S$. flexneri $\mathrm{Y} \mathrm{O}$ - and Ipa antigens in a dose-dependent manner ${ }^{108}$. The high baseline titres of serum antibodies to S. flexneri Y O- and Ipa antigens in the Vietnamese children indicate that the vaccine was seen as a booster rather than as a primary immunogen. It is not clear whether the difference in the immune responses to SC602 and SFL124 live oral Shigella vaccines in developing country populations is due to differences in the populations that participated in the clinical trials, to the inherent immunogenicity of the two strains or to disparities in the immunological assays.

\section{Shigella conjugate vaccines}

Investigators at the National Institute of Child Health and Human Development (NICHD) in the United States have proposed that in subjects immune to Shigella small amounts of serum IgG transude onto the gut surface, where they can neutralize or otherwise inactivate inocula of wild-type Shigella organisms shortly after ingestion ${ }^{109,110}$ (FIG. 3). On the basis of this concept, NICHD investigators have developed parenteral conjugate vaccines that consist of O polysaccharides derived from the LPS of relevant Shigella serotypes covalently linked to a carrier protein (Pseudomonas aeruginosa exotoxin A (PsA) or CRM9mutant diphtheria toxin). Data supporting the concept that serum IgG anti-O-antigen antibodies correlate with (and might mediate) protection come from the seroepidemiological studies of Cohen and colleagues ${ }^{111,112,131}$. Baseline serum specimens were obtained from Israeli military conscripts upon deployment to training bases where they were exposed to a high risk of shigellosis ${ }^{111,112,131}$. The rates of S. sonnei and S. flexneri 2a disease were significantly lower in individuals who had elevated non-IgM anti-O-antigen titres at baseline. Additional supporting data come from the studies of Black et al., who showed 
that North American volunteers with elevated serum IgA and IgG anti-S. sonnei-O-antigen antibodies following oral immunization with attenuated Salmonella Typhi expressing S. sonnei $\mathrm{O}$-antigen had lower infection rates when challenged with virulent $S$. sonnei in experimental challenge studies ${ }^{93}$.

A parenteral prototype NICHD vaccine that consists of S. sonnei $\mathrm{O}$ polysaccharide conjugated to PsA was shown to be well tolerated and highly immunogenic in stimulating serum IgG O-antigen antibodies in young Israeli soldiers ${ }^{113,114}$. In a randomized, controlled, double-blind Phase III efficacy trial involving several hundred Israeli soldiers, a single dose of the $S$. sonnei conjugate vaccine conferred $74 \%$ protection against $S$. sonnei diarrhoea during outbreaks on army bases ${ }^{114}$. The efficacy of the vaccine was related to the level of conjugate-induced IgG anti-O-antigen antibody.

Phase II trials assessed the clinical acceptability and immunogenicity of S. sonnei (conjugated to PsA or CRM9) and $S$. flexneri 2 a (conjugated to PsA) vaccines in Israeli children that were 4-7 and 1-4 years of age $\mathrm{e}^{115,116}$. In these paediatric studies, a two-dose immunization schedule was used, with doses spaced 6 weeks apart ${ }^{115,116}$. The first injection of each vaccine stimulated a significant (9-13-fold) rise in homologous serum IgG anti-O-antigen antibodies $^{116}$. In 1-4-year-olds, the second injection stimulated a booster response in both recipients of S. sonnei (2.9-fold) and S. flexneri 2 a (1.6-fold) conjugates; the titres remained elevated 2 years after vaccination ${ }^{116}$.

A Phase III randomized efficacy trial of S. sonnei conjugate and $S$. flexneri 2a conjugate vaccines, with each serving as a control for the other, was recently completed in paediatric subjects in Israel. The results of this trial will determine whether the $S$. sonnei conjugate vaccine can protect young children. The next generation of Shigella conjugates, based on synthetic oligosaccharides linked to proteins, offers promise for enhanced immunogenicity and diminished production costs ${ }^{117}$.

Strategies to achieve broad-spectrum protection Arguably the greatest impediment to achieving a useful Shigella vaccine is devising a strategy that can confer broad protection against a large number of epidemiologically relevant serotypes. There is widespread agreement that a global Shigella vaccine must protect against 16 serotypes and subserotypes, namely $S$. dysenteriae $1, S$. sonnei and all 14 classical S. flexneri types and subserotypes (TABLE 1; BOX 1; FIG. 2). Yet a vaccine containing so many strains or conjugates would be impractical and expensive, even if it could be formulated. To resolve this dilemma with a 'pentavalent strategy', researchers at the CVD included S. flexneri 2a, S. flexneri 3 a and S. flexneri 6 strains along with the attenuated $S$. dysenteriae 1 and $S$. sonnei strains in a multivalent vaccine, because these $3 \mathrm{~S}$. flexneri serotypes have $\mathrm{O}$-antigen group determinants that are shared by the remaining $11 \mathrm{~S}$. flexneri serotypes and subserotypes ${ }^{118}$ (BOX 1). CVD investigators have demonstrated in a guinea pig model that a multivalent vaccine containing serotypes S. flexneri $2 \mathrm{a}$ and $S$. flexneri 3 a provides an impressive level of cross-protection against other $S$. flexneri serotypes that share the common $\mathrm{O}$-antigen epitopes ${ }^{118}$.
As Ipa and VirG proteins are plasmid-encoded virulence attributes that are expressed by all virulent Shigella, irrespective of serotype, it is attractive to propose basing broad protection on stimulating serological and CMI responses to these (and perhaps to other common protein) antigens (FIG. 3). However, such responses will have to be even stronger than those elicited by wild-type infection (FIG. 3). This is because NHP challenges and epidemiological data suggest that protection due to wildtype infection does not confer serogroup-heterologous cross-protection, despite eliciting serological responses to Ipa proteins ${ }^{53}$.

\section{The way forward}

Identification of the correlates of protection is arguably the most crucial catalyst needed to accelerate the development of effective Shigella vaccines. The strongest immunizing regimens that confer protection (wildtype infection and live oral vaccines) appear to mediate protection via a confluence of immune responses. These include systemic and mucosal antibody responses to Shigella $\mathrm{O}$ - (and other) antigens, cytokines (particularly IFN- $\gamma$, secreted early as part of the innate immune response and later by specific $\mathrm{T}$ cells) and other CMI responses. Correlation of the panoply of effector immune responses elicited in subjects immunized with Shigella vaccines, exposed to wild-type Shigella and protected due to exposure to wild-type Shigella will help us identify the key immune responses that afford strong, long-lasting protection from disease. Another catalyst required to accelerate Shigella vaccine development is increased financial support for clinical trials (including challenge studies) to evaluate vaccine candidates and elucidate correlates of protection.

Although attractive candidate vaccines have entered clinical development, the road ahead presents barriers to be overcome. Whether the pentavalent strategy of the CVD can provide broad cross-protection in humans remains an important question. Also, we must document whether Shigella vaccines - live oral or parenteral conjugate - can protect vulnerable immunologically naive paediatric populations in developing countries as well as travellers from developed countries. Once vaccines have been developed that overcome the biological and epidemiological hurdles and result in licensed products, realization of the potential of vaccines as public health tools will require that yet other hurdles be surmounted, including generating political will and devising financial solutions to assure the introduction and programmatic use of vaccines in developing countries. The advent of the Global Alliance for Vaccines and Immunisation (GAVI) and its Fund, Advanced Market Commitments, the International Finance Facility for Immunisation and other innovative tools for financing the purchase of vaccines for the poorest developing countries offer tangible solutions that were unimaginable a decade ago. These innovations offer optimism that the fruits of microbiological and immunological research, such as the development of well tolerated, effective Shigella vaccines, can reach the neediest target populations. 
Shiga, K. The trend of prevention, therapy and epidemiology of dysentery since the discovery of its causative organism. N. Eng. J. Med. 215, 1205-1211 (1936).

Musings on a lifetime of study of Shigella infections by the discoverer of this pathogen.

2. Shiga, K. Über den Dysenteribacillus (Bacillus dysenteriae). Zentrablatt für Bakteriologie Parasitenkunde u. Infektionskrankheiten. Erste Parasitenkunde u. Infektionskrankheiten. Erste tierische Parasitenkunde 24, 817-818 (1898). Yabuuchi, E. Bacillus dysentericus (sic) 1897 was the first taxonomic rather than Bacillus dysenteriae 1898. Int. J. Syst. Evol. Microbiol. 52, 1041 (2002).

4. Strockbine, N. A. \& Maurelli, A. T. in Bergey's Manual of Systematic Bacteriology (eds. Brenner, D. J., Krieg, N. R. \& Staley, T. E.) 811-823 (Springer, New York, 2005)

Kotloff, K. L. et al. Global burden of Shigella infections: implications for vaccine development and implementation of control strategies. Bull. World Health Organ. 77, 651-666 (1999). A review of the epidemiological importance of Shigella infections.

6. Ferreccio, C. et al. Epidemiologic patterns of acute diarrhea and endemic Shigella infections in a poor periurban setting in Santiago, Chile. Am. J. Epidemiol. 134, 614-627 (1991).

The epidemiological study of paediatric shigellosis shows that under natural conditions of exposure, prior clinical Shigella infection confers $\sim 72 \%$ protection against subsequent disease upon exposure to a homologous serotype.

von Seidlein L. et al. A multicentre study of Shigella diarrhoea in six Asian countries: disease burden, clinical manifestations, and microbiology. Plos. Med. 3 e353 (2006)

8. Dutta, P. et al. Assessing the cause of in-patients pediatric diarrheal deaths: an analysis of hospital records. Indian Pediatr. 32, 313-321 (1995).

9. Brooks, J. T. et al. Epidemiology of sporadic bloody diarrhea in rural Western Kenya. Am. J. Trop. Med. Hyg. 68, 671-677 (2003)

10. Fulla, N., Prado, V., Duran, C., Lagos, R. \& Levine, M. M. Surveillance for antimicrobial resistance profiles among Shigella species isolated from a semirural community in the northern administrative area of Santiago, Chile. Am. J. Trop. Med. Hyg. 72 851-854 (2005)

11. Mata, L., Gangarosa, E., Caceres, A., Perera, D. \& Mejicanos, M. Epidemic Shiga bacillus dysentery in Central America. I. Etiologic investigations in Guatemala 1969. J. Infect. Dis. 122, 170-180 (1970).

Epidemiological and microbiological studies at the onset of the Central American pandemic of Shiga dysentery in the late 1960s.

12. Rahaman, M. M., Khan, M. M., Aziz, K. M. S. Islam, M. S. \& Kibriya, A. K. An outbreak of dysentery caused by Shigella dysenteriae type 1 on a Coral Island in the Bay of Bengal. J. Infect. Dis. 132, 15-19 (1975).

13. Ebright, J. R. et al. Epidemic Shiga bacillus dysentery in Central Africa. Am. J. Trop. Med. Hyg. 33 1192-1197 (1984)

14. Aragon, M., Barreto, A., Chambule, J., Noya, A. \& Tallarico, M. Shigellosis in Mozambique: the 1993 outbreak rehabilitation - a follow-up study. Trop. Doct. 25, 159-162 (1995).

15. Birmingham, M. E., Lee, L. A., Ntakibirora, M. Bizimana, F. \& Deming, M. S. A household survey of dysentery in Burundi: implications for the current pandemic in sub-Saharan Africa. Bull. World Health Organ. 75, 45-53 (1997)

16. Shane, A. L., Tucker, N. A., Crump, J. A., Mintz, E. D. \& Painter, J. A. Sharing Shigella: risk factors for a multicommunity outbreak of shigellosis. Arch. Pediatr. Adolesc. Med. 157, 601-603 (2003).

17. Mohle-Boetani, J. C. et al. Communitywide shigellosis: control of an outbreak and risk factors in child day-care centers. Am. J. Public Health. 85, 812-816 (1995).

18. Hyams, K. C. et al. Diarrheal disease during Operation Desert Shield. N. Engl. J. Med. 325, 1423-1428 (1991).

19. Levine, M. M. Immunization against bacterial diseases of the intestine. J. Pediatr. Gastroenterol. Nutr. 31, 336-355 (2000)

20. Cossart, P. \& Sansonetti, P. J. Bacterial invasion: the paradigms of enteroinvasive pathogens. Science 304 242-248 (2004).

21. Phalipon, A. \& Sansonetti, P. J. Shigella's ways of manipulating the host intestinal innate and adaptive immune system: a tool box for survival? Immunol. Cell Biol. 85, 119-129 (2007).
22. Levine, M. M. et al. Pathogenesis of Shigella dysenteriae 1 (Shiga) dysentery. J. Infect. Dis. 127 261-270 (1973).

23. DuPont, H. L., Hornick, R. B., Dawkins, A. T. Snyder, M. J. \& Formal, S. B. The response of man to virulent Shigella flexneri 2a. J. Infect. Dis. 119 296-299 (1969).

24. Herrington, D. A. et al. Studies in volunteers to evaluate candidate Shigella vaccines: further experience with a bivalent Salmonella typhi-Shigella sonnei vaccine and protection conferred by previous Shigella sonnei disease. Vaccine 8, 353-357 (1990).

25. Kotloff, K. L. et al. A modified Shigella volunteer challenge model in which the inoculum is administered with bicarbonate buffer: clinical experience and implications for Shigella infectivity. Vaccine 13 1488-1494 (1995).

Administering Shigella with bicarbonate buffer in the volunteer model assures a consistent infection rate. Volunteers who experienced shigellosis showed $\sim 70 \%$ protection against disease upon re-challenge compared with controls.

26. DuPont, H. L., Levine M. M., Hornick, R. B. \& Formal, S. B. Inoculum size in shigellosis and implications for expected mode of transmission. J. Infect. Dis. 159, 1126-1128 (1989). Reviews early volunteer studies showing that shigellosis can result from the ingestion of small inocula.

27. Levine, M. M. et al. Studies with a new generation of oral attenuated Shigella vaccine: Escherichia coli bearing surface antigens of Shigella flexneri. J. Infect. Dis. 136, 577-582 (1977).

An $E$. coli hybrid expressing $S$. flexneri 2a O-antigens failed to protect against $S$. flexneri 2 a challenge.

28. Coster, T. S. et al. Vaccination against shigellosis with attenuated Shigella flexneri 2a strain SC602. Infect. Immun. 67, 3437-3443 (1999).

Albeit a reactogenic strain, a single dose of SC602 protected volunteers against challenge with wildtype $S$. flexneri $2 a$.

29. Formal, S. B. et al. Protection of monkeys against experimental shigellosis with a living attenuated oral polyvalent dysentery vaccine. J. Bacteriol. 92, 17-22 (1966).

30. Formal, S. B., Maenza, R. M., Austin, S. \& LaBrec, E. H. Failure of parenteral vaccines to protect monkeys against experimental shigellosis. Proc. Soc. Exp. Biol. 125, 347-349 (1967)

31. Formal, S. B. et al. Effect of prior infection with virulent Shigella flexneri 2a on the resistance of monkeys to subsequent infection with Shigella sonnei. J. Infect. Dis. 164, 533-537 (1991)

NHPs that had recovered from $S$. flexneri $2 a$ challenge were protected against re-challenge with $S$. flexneri 2 a but not against challenge with heterologous serotype $S$. sonnei.

32. Rout, W. R., Formal, S. B., Giannella, R. A. \& Dammin, G. J. Pathophysiology of Shigella diarrhea in the rhesus monkey: intestinal transport, morphological, and bacteriological studies. Gastroenterol. 68 270-278 (1975).

Demonstrated that NHPs experiencing Shigella diarrhoea for some hours before the onset of dysentery had net jejunal secretion, implying the action of an enterotoxin.

33. Kinsey, M. D., Formal, S. B., Dammin, G. J. \& Giannella, R. A. Fluid and electrolyte transport in rhesus monkeys challenged intracecally with Shigella flexneri 2a. Infect. Immun. 14, 368-371 (1976).

34. Fasano, A. et al. Shigella enterotoxin 1: an enterotoxin of Shigella flexneri 2a active in rabbit small intestine in vivo and in vitro. J. Clin. Invest. 95, 2853-2861 (1995).

Identification and characterization of ShET1

35. Fasano, A., Noriega, F. R., Liao, F. M., Wang, W. \& Levine, M. M. Effect of Shigella enterotoxin 1 (ShET1) on rabbit intestine in vitro and in vivo. Gut $\mathbf{4 0}$, 505-511 (1997)

36. Nataro, J. P. et al. Identification and cloning of a novel plasmid-encoded enterotoxin of enteroinvasive Escherichia coli and Shigella strains. Infect. Immun. 63, 4721-4728 (1995)

Discovery of plasmid-encoded ShET2.

37. Noriega, F. R., Liao, F. M., Formal, S. B., Fasano, A. \& Levine, M. M. Prevalence of Shigella enterotoxin 1 among Shigella clinical isolates of diverse serotypes. J. Infect. Dis. 172, 1408-1410 (1995).

38. Nie, H. et al. Complete genome sequence of Shigella flexneri $5 \mathrm{~b}$ and comparison with Shigella flexneri $2 \mathrm{a}$ BMC Genomics 7, 173 (2006).
39 Yavzori, M. Cohen, D \& Orr N. Prevalence of the genes for Shigella enterotoxins 1 and 2 among clinical isolates of Shigella in Israel. Epidemiol. Infect. 128 533-535 (2002)

40. Roy, S., Thanasekaran, K., Dutta Roy, A. R. \& Sehgal, S. C. Distribution of Shigella enterotoxin genes and secreted autotransporter toxin gene among diverse species and serotypes of Shigella isolated from Andaman Islands, India. Trop. Med. Int. Health 11. 1694-1698 (2006).

41. Niyogi, S. K., Vargas, M. \& Vila, J. Prevalence of the sat, set and sen genes among diverse serotypes of Shigella flexneri strains isolated from patients with acute diarrhoea. Clin. Microbiol. Infect. 10, 574-576 (2004).

42. DuPont, H. L. et al. Immunity in shigellosis. II. Protection induced by oral live vaccine or primary infection. J. Infect. Dis. 125, 12-16 (1972).

43. Oberhelman, R. et al. Prospective study of systemic and mucosal immune responses in dysenteric patients to specific Shigella invasion plasmid antigens and lipopolysaccharides. Infect. Immun. 59, 2341-2350 (1991)

44. Islam, D., Wretlind, B., Ryd, M., Lindberg, A. A. \& Christensson, B. Immunoglobulin subclass distribution and dynamics of Shigella-specific antibody responses in serum and stool samples in shigellosis. Infect. Immun. 63, 2054-2061 (1995).

45. Azim, T. et al. Lipopolysaccharide-specific antibodies in plasma and stools of children with Shigellaassociated leukemoid reaction and hemolytic-uremic syndrome. Clin. Diagn. Lab. Immunol. 3, 701-705 (1996).

46. Orr, N., Robin, G., Lowell, G. \& Cohen, D. Presence of specific immunoglobulin A-secreting cells in peripheral blood after natural infection with Shigella sonnei. J. Clin. Microbiol. 30, 2165-2168 (1992).

47. Cohen, D. et al. Detection of antibodies to Shigella lipopolysaccharide in urine after natural Shigella infection or vaccination. Clin. Diagn. Lab. Immunol. 3 451-455 (1996)

48. Van de Verg, L. et al. Specific immunoglobulin A-secreting cells in peripheral blood of human following oral immunization with a bivalent Salmonella typhi-Shigella sonnei vaccine or infection by pathogenic $S$. sonnei. Infect. Immun. $\mathbf{5 8}$ 2002-2004 (1990).

49. Kotloff, K. L. et al. Safety, immunogenicity, and efficacy in monkeys and humans of invasive Escherichia coli K-12 hybrid vaccine candidates expressing Shigella flexneri 2a somatic antigen. Infect. Immun. 60, 2218-2224 (1992).

50. Kotloff, K. L. et al. Evaluation of the safety, immunogenicity and efficacy in healthy adults of four doses of live oral hybrid Escherichia coli-Shigella flexneri 2 a vaccine strain EcSf2a-2. Vaccine 13 495-502 (1995).

51. Levine, M. M., Gangarosa, E. J., Werner, M. \& Morris, J. G. Shigellosis in custodial institutions. III. Prospective clinical and bacteriologic surveillance of children vaccinated with oral attenuated Shigella vaccine. J. Pediatr. 84, 803-806 (1974).

52. Tacket, C. O. et al. Efficacy of bovine milk immunoglobulin concentrate in preventing illness after Shigella flexneri challenge. Am. J. Trop. Med. Hyg. 47 276-283 (1992).

Cow's milk immunoglobulin containing high titres of anti-Shigella antibodies protected volunteers against diarrhoeal illness following challenge with S. flexneri $2 \mathrm{a}$.

53. Oaks, E. V., Hale, T. L. \& Formal, S. B. Serum immune response to Shigella protein antigens in rhesus monkeys and humans infected with Shigella spp. Infect. Immun. 53, 57-63 (1986).

54. Van de Verg, L. L., Herrington, D. A., Boslego, J., Lindberg, A. A. \& Levine, M. M. Age-specific prevalence of serum antibodies to the invasion plasmid and lipopolysaccharide antigens of Shigella species in Chilean and North American populations. J. Infect. Dis. 166, 158-161 (1992).

55. Raqib, R. et al. Dissociation between cytokine mRNA expression and protein production in shigellosis. Eur. J. Immunol. 26, 1130-1138 (1996).

56. Raqib, R. et al. Persistence of local cytokine production in shigellosis in acute and convalescent stages. Infect. Immun. 63, 289-296 (1995).

57. Raqib, R., Wretlind, B., Andersson, J. \& Lindberg, A. A Cytokine secretion in acute shigellosis is correlated to disease activity and directed more to stool than to plasma. J. Infect. Dis. 171, 376-384 (1995). 
58. Raqib, R., Ljungdahl, A., Lindberg, A. A., Andersson, U. $\S$ Andersson, J. Local entrapment of interferon $\gamma$ in the recovery from Shigella dysenteriae type 1 infection. Gut 38, 328-336 (1996)

59. Raqib, R. et al. Delayed and reduced adaptive humoral immune responses in children with shigellosis compared with in adults. Scand. J. Immunol. 55 414-423 (2002)

60. Samandari, T. et al. Production of IFN- $\gamma$ and IL-10 to Shigella invasins by mononuclear cells from volunteers orally inoculated with a shiga toxin-deleted Shigella dysenteriae type 1 strain. J. Immunol. 164 2221-2232 (2000)

61. Islam, D., Bardhan, P. K., Lindberg, A. A. \& Christensson, B. Shigella infection induces cellular activation of $\mathrm{T}$ and $\mathrm{B}$ cells and distinct species-related changes in peripheral blood lymphocyte subsets during the course of the disease. Infect. Immun. 63 , 2941-2949 (1995)

62. Islam, D., Wretlind, B., Lindberg, A. A. \& Christensson, B. Changes in the peripheral blood T-cell receptor $\mathrm{V} \beta$ repertoire in vivo and in vitro during shigellosis. Infect. Immun. 64, 1391-1399 (1996).

63. World Health Organization. Future needs and directions for Shigella vaccines. Wkly Epidemiol. Rec. 81, 51-58 (2006).

64. Mel, D. M., Terzin, A. L. \& Vuksic, L. Studies on vaccination against bacillary dysentery. 1 . Immunization of mice against experimental Shigella infection. Bull. World Health Organ. 32, 633-636 (1965)

65. Mel, D. M., Papo, R. G., Terzin, A. L. \& Vuksic, L. Studies on vaccination against bacillary dysentery. 2 Safety tests and reactogenicity studies on a live dysentery vaccine intended for use in field trials. Bull. World Health Organ 32, 637-645 (1965).

66. Sereny, B. Experimental keratoconjunctivitis shigellosa. Acta Microbiol. Acad. Sci. Hung. 4, 367-376 (1957).

67. Mel, D. M., Terzin, A. L. \& Vuksic, L. Studies on vaccination against bacillary dysentery. 3 . Effective oral immunization against Shigella flexneri 2a in a field trial. Bull. World Health Organ. 32, 647-655 (1965).

68 Mel, D. M. Arsic, B. L., Nikolic, B. D. \& Radovanovic, M. L. Studies on vaccination against bacillary dysentery. 4. Oral immunization with live monotypic and combined vaccines. Bull. World Health Organ. 39, 375-380 (1968).

Multivalent oral Shigella vaccine protected children against shigellosis in a controlled field trial.

69. Mel, D. M., Gangarosa, E. J., Radovanovic, M. L., Arsic, B. L. \& Litvinjenko, S. Studies on vaccination against bacillary dysentery. 6 . Protection of children by oral immunization with streptomycin-dependent Shigella strains. Bull. World Health Organ. 45, 457-464 (1971)

Oral SmD Shigella vaccine conferred protection for less than 1 year; a single oral booster extended protection.

70. Mel, D. M., Arsic, B. L., Radovanovic, M. L. \& Litvinjenko, S. Live oral Shigella vaccine: vaccination schedule and the effect of booster dose. Acta Microbiol. Acad. Sci. Hung. 21, 109-114 (1974).

71. DuPont, H. L. et al. Immunity in shigellosis. I. Response of man to attenuated strains of Shigella. J. Infect. Dis. 125, 5-11 (1972).

72. Levine, M. M. et al. Shigellosis in custodial institutions II. Clinical, immunologic and bacteriologic response of institutionalized children to oral attenuated Shigella vaccines. Am. J. Epidemiol. 96, 40-49 (1972).

73. Levine, M. M. et al. Shigellosis in custodial institutions. IV. In vivo stability and transmissibility of oral attenuated streptomycin-dependent Shigella vaccines. J. Infect. Dis. 131, 704-707 (1975)

74. Levine, M. M., Gangarosa, E. J., Barrow, W. B. \& Weiss, C. F. Shigellosis in custodial institutions. $V$. Effect of intervention with streptomycin-dependent Shigella sonnei vaccine in an institution with endemic disease. Am. J. Epidemiol. 104, 88-92 (1976)

75. Damjanovic, V. Freeze-drying of live oral streptomycin dependent mutant Shigella vaccines: survival of streptomycin-dependent Shigella flexneri $2 \mathrm{a}$ after freeze-drying. Cryobiology 9, 565-568 (1972).

76. Meitert, T. et al. Prophylaxie de la dysenterie bacillaire par le vaccin vivant antidysentérique dans une collectivité d'enfants neuropsychiques. Arch. Roum Path Exp Microbiol 32 35-44 (1973)

77. Meitert, T., Pencu, E., Ciudin, L. \& Tonciu, M. Vaccine strain Sh. flexneri T32-Istrati. Studies in animals and in volunteers. Antidysentery immunoprophylaxis and immunotherapy by live vaccine Vadizen (Sh. flexneri T32-Istrati). Arch. Roum. Pathol. Exp. Microbiol. 43 251-278 (1984).
78. Bingrui, W. Study on the effect of oral immunization of T32-Istrati strain against bacillary dysentery in field trials. Arch. Roum. Pathol. Exp. Microbiol. 43, 285-289 (1984)

79. Venkatesan, M., Fernandez Prada, C., Buysse, J. M., Formal, S. B. \& Hale, T. L. Virulence phenotype and genetic characteristics of the T32- ISTRATI Shigella flexneri 2 a vaccine strain. Vaccine $9,358-363$ (1991).

80. Tu, G. et al. Double-blind field trial of oral live $\mathrm{F}_{2}$ Sonnei (FS) dysentery vaccine. J. Biol. Prod. 12, 178-180 (1999).

81. Formal, S. B., LaBrec, E. H., Palmer, A. \& Falkow, S. Protection of monkeys against experimental shigellosis with attenuated vaccines. J. Bacteriol. 90, 63-68 (1965).

82. Falkow, S., Schneider, H., Baron, L. S. \& Formal, S. B. Virulence of Escherichia-Shigella genetic hybrids for the guinea pig. J. Bacteriol. 86, 1251-1258 (1963).

83. Li, A. et al. Safety and immunogenicity of the live oral auxotrophic Shigella flexneri SFL124 in adult Vietnamese volunteers. Vaccine 11, 180-189 (1993)

84. Lindberg, A. A., Karnell, A. \& Stocker, B. A. D. in Aromatic-Dependent Shigella Strains as Oral Live Vaccines (eds. Woodrow, G. C. \& Levine, M. M.) 677-687 (Marcel Dekker, New York, 1990).

85. Karnell, A. et al. Safety and immunogenicity study of the auxotrophic Shigella flexneri 2a vaccine SFL1070 with a deleted $a r o D$ gene in adult Swedish volunteers. Vaccine 13, 88-89 (1995).

86. Kotloff, K. L. et al. Safety, immunogenicity, and transmissibility in humans of CVD 1203, a live oral Shigella flexneri 2 a vaccine candidate attenuated by deletions in aroA and virG. Infect. Immun. 64 4542-4548 (1996).

87. Kotloff, K. L. et al. Shigella flexneri 2a strain CVD 1207 , with specific deletions in virG, sen, set, and guaBA, is highly attenuated in humans. Infect. Immun. 68, 1034-1039 (2000)

88. Kotloff, K. L. et al. Deletion in the Shigella enterotoxin genes further attenuates Shigella flexneri 2a bearing guanine auxotrophy in a Phase 1 trial of CVD 1204 and CVD 1208. J. Infect. Dis. 190, 1745-1754 (2004)

This randomized, double-blind clinical trial confirmed that ShET1 and ShET2 cause diarrhoea.

89. Katz, D. E. et al. Two studies evaluating the safety and immunogenicity of a live, attenuated Shigella flexneri 2 a vaccine (SC602) and excretion of vaccine organisms in North American volunteers. Infect. Immun. 72, 923-930 (2004)

90. Noriega, F. R. et al. Construction and characterization of attenuated $\Delta$ aroA $\Delta$ virG Shigella flexneri 2a strain CVD 1203, a prototype live oral vaccine. Infect. Immun. 65, 5168-5172 (1994).

91. Noriega, F. R. et al. Engineered $\Delta$ guaB-A, $\Delta$ virG Shigella flexneri 2a strain CVD 1205: construction, safety, immunogenicity and potential efficacy as a mucosal vaccine. Infect. Immun. 64, 3055-3061 (1996).

92. Hartman, A. B. \& Venkatesan, M. M. Construction of a stable attenuated Shigella sonnei $\Delta v i r G$ vaccine strain, WRSS 1 , and protective efficacy and immunogenicity in the guinea pig keratoconjunctivitis model. Infect. Immun 66, 4572-4576 (1998).

93. Black, R. E. et al. Prevention of shigellosis by a Salmonella typhi-Shigella sonnei bivalent vaccine. J. Infect. Dis. 155, 1260-1265 (1987)

Two pilot lots of this live vector vaccine conferred protection against challenge with $S$. sonnei, whereas other lots did not.

94. Kotloff, K. et al. Phase 1 evaluation of a vriG deleted Shigella sonnei live, attenuated vaccine (strain WRSS1) in healthy adult volunteers. Infect. Immun. 70, 2016 2021 (2002).

95. Orr, N. et al. Community-based safety, immunogenicity, and transmissibility study of the Shigella sonnei WRSS 1 vaccine in Israeli volunteers. Infect. Immun. 73 8027-8032 (2005)

\section{An engineered $S$. sonnei strain was mildly}

reactogenic and moderately immunogenic in adults.

96. Newland, J. W., Hale, T. L. \& Formal, S. B. Genotypic and phenotypic characterization of an aroD deletionattenuated Escherichia coli K12-Shigella flexneri hybrid vaccine expressing $S$. flexneri 2 a somatic antigen. Vaccine 10, 766-776 (1992).

97. Formal, S. B. et al. Construction of a potential bivalent vaccine strain: introduction of Shigella sonnei form I antigen genes into the galE Salmonella typhi Ty21a typhoid vaccine strain. Infect. Immun. 34, 746-750 (1981)
98. Tramont, E. C. et al. Safety and antigenicity of typhoidShigella sonnei vaccine (strain 5076-1C). J. Infect. Dis. 149, 133-136 (1984).

99. Seid, R. C. et al. Unusual lipopolysaccharide antigens of a Salmonella typhi oral vaccine strain expressing the Shigella sonnei form I antigen. J. Biol. Chem. 259, 9028-9034 (1984).

100. Schultz, C. L. et al. Cell wall structures which may be important for successful immunization with Salmonella-Shigella hybrid vaccines. Vaccine 8 115-120 (1990).

101. Hartman, A. B., Ruiz, M. M. \& Schultz, C. L. Molecular analysis of variant plasmid forms of a bivalent Salmonella typhi-Shigella sonnei vaccine strain. J. Clin. Microbiol. 29, 27-32 (1991).

102. Hartman, A. B., Powell, C. J., Schultz, C. L., Oaks, E. V. \& Eckels, K. H. Small-animal model to measure efficacy and immunogenicity of Shigella vaccine strains. Infect. Immun. 59, 4075-4083 (1991).

103. Xu, D. Q., Cisar, J. O., Ambulos, J. N., Jr, Burr, D. H. \& Kopecko, D. J. Molecular cloning and characterization of genes for Shigella sonnei form I O polysaccharide: proposed biosynthetic pathway and stable expression in a live Salmonella vaccine vector. Infect. Immun. 70, 4414-4423 (2002)

104. John, T. J. Immunisation against polioviruses in developing countries. Rev. Med. Virol. 3, 149-160 (1993).

105. Hanlon, P. et al. Trial of an attenuated bovine rotavirus vaccine (RIT 4237) in Gambian infants. Lancet 1 , 1342-1345 (1987)

106. Suharyono et al. Safety and immunogenicity of singledose live oral cholera vaccine CVD 103-HgR in 5-9year-old Indonesian children. Lancet 340, 689-694 (1992)

107. Hallander, H. O. et al. Calibrated serological techniques demonstrate significant different serum response rates to an oral killed cholera vaccine between Swedish and Nicaraguan children. Vaccine 21, 138-145 (2002)

108. Li, A. et al. Immune responses in Vietnamese children after a single dose of the auxotrophic, live Shigella flexneri $Y$ vaccine strain SFL124. J. Infect. 28, 11-23 (1994).

An aroD mutant $S$. flexneri Y strain stimulated ASC responses in Vietnamese children.

109. Robbins, J. B., Chu, C. \& Schneerson, R. Hypothesis for vaccine development: protective immunity to enteric diseases caused by non-typhoidal Salmonellae and Shigellae may be conferred by serum IgG antibodies to the O-specific polysaccharides of their lipopolysaccharides. Clin. Infect. Dis. 15, 346-351 (1992).

110. Chowers, Y. et al. Specific polysaccharide conjugate vaccine-induced $\lg \mathrm{G}$ antibodies prevent invasion of Shigella into Caco-2 cells and may be curative. Proc Natl Acad. Sci. USA 104, 2396-2401 (2007).

111. Cohen, D., Green, M. S., Block, C., Slepon, R. \& Ofek, I. Prospective study of the association between serum antibodies to lipopolysaccharide $\mathrm{O}$ antigen and the attack rate of shigellosis. J. Clin. Microbiol. 29 386-389 (1991)

Soldiers with elevated titres of Shigella O-antigen antibodies had lower attack rates of shigellosis tha sero-negative soldiers upon natural exposure in the field.

112 Cohen, D., Green, M. S., Block, C., Rouach, T. \& Ofek, I. Serum antibodies to lipopolysaccharide and natural immunity to shigellosis in an Israeli military population. J. Infect. Dis. 157, 1068-1071 (1988).

113. Cohen, D. et al. Safety and immunogenicity of investigational Shigella conjugate vaccines in Israeli volunteers. Infect. Immun. 64, 4074-4077 (1996).

14. Cohen, D. et al. Double-blind vaccine-controlled randomised efficacy trial of an investigational Shigella sonnei conjugate vaccine in young adults. Lancet 349 155-159 (1997)

An $S$. sonnei conjugate vaccine protected young adult soldiers against $S$. sonnei shigellosis under conditions of natural exposure in the field.

115. Ashkenazi, S. et al. Safety and immunogenicity of Shigella sonnei and Shigella flexneri 2a O-specific polysaccharide conjugates in children. J. Infect. Dis. 179, 1565-1568 (1999)

116. Passwell, J. H. et al. Safety and immunogenicity of Shigella sonnei-CRM9 and Shigella flexneri type $2 a$ rEPAsucc conjugate vaccines in one- to four-year-old children. Pediatr. Infect. Dis. J. 22, 701-706 (2003).

117. Phalipon, A. et al. Characterization of functional oligosaccharide mimics of the Shigella flexneri serotype 2a O-antigen: implications for the development of a chemically defined glycoconjugate vaccine. J. Immunol. 176, 1686-1694 (2006) 
118. Noriega, F. R. et al. Strategy for cross-protection among Shigella flexneri serotypes. Infect. Immun. 67, 782-788 (1999).

In the guinea pig keratoconjunctivitis model, a bivalent mucosal vaccine consisting of attenuated $S$. flexneri $2 \mathrm{a}$ and $S$. flexneri 3 a strains conferred significant cross-protection against challenge with multiple other $S$. flexneri subserotypes.

119. Lindberg, A. A., Karnell, A. \& Weintraub, A. The lipopolysaccharide of Shigella bacteria as a virulence factor. Rev. Infect. Dis. 13 (Suppl. 4), S279-S284 (1991).

120. West, N. P. et al. Optimization of virulence functions through glucosylation of Shigella LPS. Science. $\mathbf{3 0 7}$ 1313-1317 (2005)

121. Carlin, N. I., Wehler, T. \& Lindberg, A. A. Shigella flexneri O-antigen epitopes: chemical and immunochemical analyses reveal that epitopes of type III and group 6 antigens are identical. Infect. Immun. $53,110-115$ (1986)

122. Carlin, N. I. et al. Use of monoclonal antibodies to type Shigella flexneri in Bangladesh. J. Clin. Microbiol. 27, 1163-1166 (1989).

123. Feng, L. et al. Structural and genetic evidence that the Escherichia coli O148 O antigen is the precurso of the Shigella dysenteriae type 10 antigen and identification of a glucosyltransferase gene. Microbiology 153, 139-147 (2007).

124. Fries, L. F. et al. Safety and immunogenicity of a proteosome - Shigella flexneri 2a lipopolysaccharide vaccine administered intranasally to healthy adults. Infect. Immun. 69, 4545-4553 (2001).

125. McKenzie, R. et al. Safety and immunogenicity of an oral, inactivated, whole-cell vaccine for Shigella sonnei: preclinical studies and a Phase I trial. Vaccine 24, 3735-3745 (2006).

126. Oaks, E. V. \& Turbyfill, K. R. Development and evaluation of a Shigella flexneri $2 \mathrm{a}$ and $\mathrm{S}$. sonne bivalent invasin complex (Invaplex) vaccine. Vaccine 24, 2290-2301 (2006).
127. Levenson, V. et al. Protective ribosomal preparation from Shigella sonnei as a parenteral candidate vaccine. Infect. Immun. 59, 3610-3618 (1991).

128. D'Hauteville, H. et al. Two $m s b B$ genes encoding maximal acylation of lipid $A$ are required for invasive Shigella flexneri to mediate inflammatory rupture and destruction of the intestinal epithelium. J. Immunol. 168, 5240-5251 (2002).

129. Prado, V. et al. Population-based study of the incidence of Shigella diarrhea and causative serotypes in Santiago, Chile. Pediatr. Infect. Dis. J. 18, 500-505 (1999).

130. Ahmed, S. F. et al. Epidemiology and genetic characterization of Shigella flexneri strains isolated from three paediatric populations in Egypt (2000-2004). Epidemiol. Infect. 134, 1237-1248 (2006)

131. Cohen, D., Green, M. S., Block, C., Slepon, R. \& Lerman, Y. Natural immunity to shigellosis in two groups with different previous risks of exposure to Shigella is only partly expressed by serum antibodies to lipopolysaccharide. J. Infect. Dis. 165, 785-787 (1992)

\section{Acknowledgements}

This paper includes work funded, in part, by the National Institute of Allergy and Infectious disease (NIAID), National Institutes of Health (NIH), Department of Health and Human Services (DHHS) federal research grants and contracts N01 Al25461, R01 Al029471 and U54 Al57168 (to M.L.), R01 AI57927 and N01 AI30028 (to M.S.) and R01 Al059223 (to E.B.) and a grant from the Bill and Melinda Gates Foundation (to M.L.).

Competing interests statement

The authors declare no competing financial interests.
DATABASES

The following terms in this article are linked online to: Entrez Gene: http://www.ncbi.nlm.nih.gov/entrez/query. fcgi?db=gene

virG

Entrez Genome Project: http://www.ncbi.nlm.nih.gov/ entrez/query.fcgi?CMD=search\&DB=genomeprj Shigella dysenteriae | Shigella flexneri| Shigella sonnei UniProtKB: http://ca.expasy.org/sprot IFN- $\gamma \mid$ IL-10|TNF- $\alpha$

\section{FURTHER INFORMATION}

Eileen Barry's homepage: http://medschool.umaryland.edu/ CVD/Fac_Res_Interest.asp?id=38

Karen Kotloff's homepage: http://medschool.umaryland. edu/facultyresearchprofile/viewprofile.aspx?id=800

Marcela Pasetti's homepage: http://medschool.umaryland. edu/facultyresearchprofile/viewprofile.aspx?id=4848

Marcelo Sztein's homepage: http://medschool.umaryland. edu/CVD/Fac_Res_Interest.asp?id=52

Myron Levine's homepage: http://medschool.umaryland. edu/facultyresearchprofile/viewprofile.aspx?id=723 Advanced Market Commitments for Vaccines: http//www.vaccineamc.org/about/index.html

Center for Vaccine Development:

http://medschool.umaryland.edu/CVD Global Alliance for Vaccines and Immunisation: http://www.gavialliance.org

International Finance Facility for Immunisation: http://www.iff-immunisation.org National Institute of Child Health and Human Development: http://www.nichd.nih.gov Walter Reed Army Institute of Research: http://wrair-www.army.mil Access to this links box is available online. 